\title{
Closed-loop Identification Revisited
}

\author{
Urban Forssell and Lennart Ljung \\ Department of Electrical Engineering \\ Linkping University, S-581 83 Linkping, Sweden \\ WWW: http://www. control.isy.liu.se \\ Email: ufo@isy.liu.se, ljung@isy.liu.se
}

1997-06-09

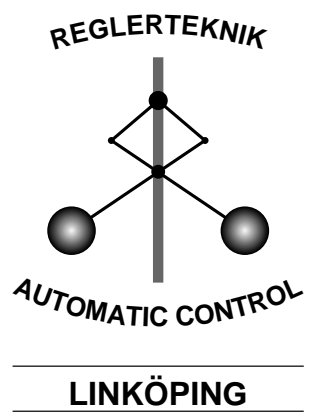

Report no.: LiTH-ISY-R-1959

Submitted to Automatica

Technical reports from the Automatic Control group in Linkping are available by anonymous ftp at the address 130.236.20.24 (ftp.control.isy.liu.se). This report is contained in the compressed postscript file 1959.ps.Z. 


\title{
Closed-loop Identification Revisited ${ }^{\star}$
}

\author{
Urban Forssell and Lennart Ljung \\ Department of Electrical Engineering, Linköping University, S-581 83 Linköping, \\ Sweden
}

\begin{abstract}
In this contribution we study the statistical properties of a number of closed-loop identification methods and parameterizations. A focus will be on asymptotic variance expressions for these methods. By studying the asymptotic variance for the parameter vector estimates we show that indirect methods fail to give better accuracy than the direct method. Some new results for the bias distribution of the direct method will be presented and we also show how different methods correspond to different parameterizations and how direct and indirect identification can be linked together via the noise model. In addition, analysis of a new method for closed-loop identification shows that it allows fitting the model to data with arbitrary frequency weighting under quite general conditions.
\end{abstract}

Key words: System identification; Closed-loop identification; Prediction error methods

\section{Introduction}

The goal in system identification is to determine a good model of a given system from observed data. Correlation and spectral analysis, instrumental variables and prediction error methods are classical examples of identification methods. More recently the so called subspace identification methods have been introduced. In the open-loop case all these methods generally work well and produce good models of the system.

This is not the case in closed-loop identification and it is well known that, e.g., spectral analysis, instrumental variable methods and the subspace methods give erroneous results when applied directly to closed-loop data.

ऋ This paper was not presented at any IFAC meeting. Corresponding author U. Forssell. Tel. +46-13-282226. Fax +46-13-282622. E-mail ufo@isy.liu.se.

Preprint submitted to Elsevier Preprint 20 November 1997 


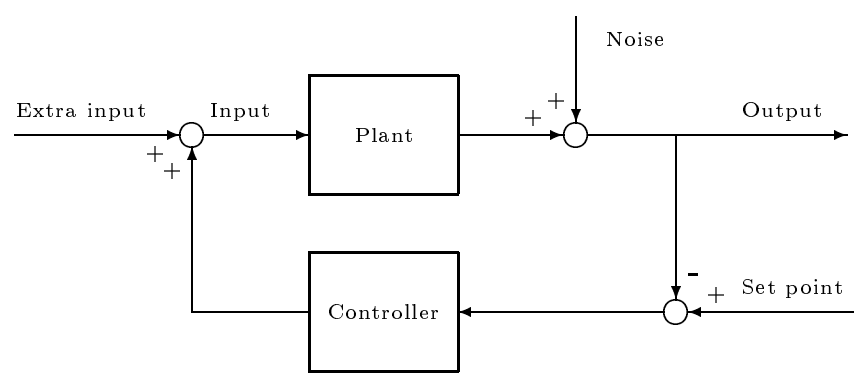

Fig. 1. A closed-loop system

The fundamental problem with closed-loop data is the correlation between the un-measurable noise and the input. Consider the system in Fig. 1. It is clear that whenever the feedback controller is not identically zero, the input and the noise will be correlated. Due to this correlation, the resulting estimate will typically be biased, as shown in the following example (of well-known character).

Example 1 Suppose we want to identify the system

$$
y(t)=B(q) u(t)+v(t)=b_{1} u(t-1)+\cdots+b_{n} u(t-n)+v(t)
$$

using a linear regression model

$$
y(t)=\varphi^{T}(t) \theta+v(t)
$$

where

$$
\theta=\left[b_{1} \ldots b_{n}\right]^{T} \text { and } \varphi(t)=[u(t-1) \ldots u(t-n)]^{T}
$$

The least-squares estimate of the parameter vector $\theta$ based on $N$ data points is given by

$$
\begin{aligned}
\hat{\theta}_{N} & =\left[\frac{1}{N} \sum_{t=1}^{N} \varphi(t) \varphi^{T}(t)\right]^{-1} \frac{1}{N} \sum_{t=1}^{N} \varphi(t) y(t) \\
& =\theta+\left[\frac{1}{N} \sum_{t=1}^{N} \varphi(t) \varphi^{T}(t)\right]^{-1} \frac{1}{N} \sum_{t=1}^{N} \varphi(t) v(t)
\end{aligned}
$$

We know that, under mild conditions, $\hat{\theta}_{N} \rightarrow \theta^{*}$ w. p. 1 where

$$
\theta^{*}=\lim _{N \rightarrow \infty} E \hat{\theta}_{N}=\theta+\left[\bar{E} \varphi(t) \varphi^{T}(t)\right]^{-1} \bar{E} \varphi(t) v(t)
$$

It is obvious that, for consistency, we need $\bar{E} \varphi(t) v(t) \equiv 0$, i.e. the noise must be uncorrelated with the input. In closed loop this is not the case and the results will hence be biased. 
Despite these problems, performing identification experiments under output feedback (i.e. in closed loop) can be advantageous. In "identification for control" the objective is to achieve a model that is suited for robust control design (see, e.g., $[3,9,19]$ ). Thus one has to tailor the experiment and preprocessing of data so that the model is reliable in regions where the design process does not tolerate significant uncertainties. The use of closed-loop experiments has been a prominent feature in these approaches. Other reasons for using closed-loop experiments might be that the plant is unstable, or that it has to be controlled for production economic or safety reasons, or that it contains inherent feedback mechanisms.

Historically there has been a substantial interest in both special identification techniques for closed-loop data, and for analysis of existing methods when applied to such data. One of the earliest results was given by Akaike [1] who analyzed the effect of feedback loops in the system on correlation and spectral analysis. In the seventies there was a very active interest in questions concerning closed-loop identification, as summarized in the survey paper [6], followed by [2]. Up to this point much of the attention had been directed towards identifiability problems. With the increasing interest in model based control, closed-loop identification has again gained a lot of attention. A main issue has then been the ability to shape the bias distribution in a control-relevant way. The surveys [4] and [16] cover most of the results along this line of research.

It is the purpose of the present paper to "revisit" the area of closed-loop identification, to put some of the new results and methods into perspective, and to give a status report of what can be done and what cannot. In the course of this exposé, some new results will also be generated.

The rest of the paper is organized as follows. Next, in Section 2 we formalize the assumptions we make regarding the system and we also introduce some notation. Section 3 contains a characterization of the basic assumptions that can be made regarding the nature of the feedback. This leads to a classification of all closed-loop identification methods into, so called, direct, indirect, and joint input-output methods. In Section 4 we discuss the direct approach. First we give some background material and present the underlying ideas with this approach. We then study the statistical properties of the resulting estimates, we characterize the bias distribution and give asymptotic variance expressions. Sections 5 and 6 contain a similar treatment of the indirect and joint inputoutput approaches, respectively. Finally, in Section 7 we summarize the basic issues on closed-loop identification. 


\section{Preliminaries}

In many cases we will not need to know the feedback mechanism, but for some of the analytic treatment we shall work with the following linear output feedback setup: The true system is

$$
\begin{aligned}
& y(t)=G_{0}(q) u(t)+v(t) \\
& v(t)=H_{0}(q) e(t)
\end{aligned}
$$

Here $\{e(t)\}$ is white noise with variance $\lambda_{0}$. The regulator (controller) is

$$
u(t)=r(t)-F_{y}(q) y(t)
$$

The reference signal $\{r(t)\}$ is assumed independent of the noise $\{e(t)\}$. We also assume that the regulator stabilizes the system and that either $G_{0}(q)$ or $F_{y}(q)$ contains a delay so that the closed-loop system is well defined.

The input can be written as

$$
u(t)=S_{0}(q) r(t)-F_{y}(q) S_{0}(q) v(t)
$$

where $S_{0}(q)$ is the sensitivity function,

$$
S_{0}(q)=\frac{1}{1+F_{y}(q) G_{0}(q)}
$$

The closed-loop system is

$$
y(t)=G_{0}(q) S_{0}(q) r(t)+S_{0}(q) v(t)
$$

With

$$
G_{c l, 0}(q)=G_{0}(q) S_{0}(q) \text { and } H_{c l, 0}(q)=S_{0}(q) H_{0}(q)
$$

we can rewrite $(3)$ as

$$
\begin{aligned}
y(t) & =G_{c l, 0}(q) r(t)+v_{c l}(t) \\
v_{c l}(t) & =H_{c l, 0}(q) e(t)
\end{aligned}
$$

To reduce the notational burden we will from here on suppress the arguments $q, \omega, e^{i \omega}$ and $t$ whenever there is no risk of confusion.

The spectrum of the input is (cf. (2))

$$
\Phi_{u}=\left|S_{0}\right|^{2} \Phi_{r}+\left|F_{y}\right|^{2}\left|S_{0}\right|^{2} \Phi_{v}
$$


where $\Phi_{r}$ is the spectrum of the reference signal and $\Phi_{v}=\left|H_{0}\right|^{2} \lambda_{0}$ the noise spectrum. We shall denote the two terms

$$
\begin{aligned}
& \Phi_{u}^{r}=\left|S_{0}\right|^{2} \Phi_{r} \\
& \Phi_{u}^{e}=\left|F_{y}\right|^{2}\left|S_{0}\right|^{2} \Phi_{v}
\end{aligned}
$$

Since we mainly will study prediction error methods the following definitions regarding model parameterization will be convenient: Consider the model set

$$
\begin{gathered}
\mathcal{M}=\{\mathcal{M}(\theta): y(t)=G(q, \theta) u(t)+H(q, \theta) e(t), \\
\left.\theta \in D_{\mathcal{M}} \subset \mathbb{R}^{d}\right\}
\end{gathered}
$$

Here $d=\operatorname{dim}(\theta)$. We say that the true system is contained in the model set if, for some $\theta_{0} \in D_{\mathcal{M}}$,

$$
G\left(q, \theta_{0}\right)=G_{0}(q), H\left(q, \theta_{0}\right)=H_{0}(q)
$$

This will also be written $\mathcal{S} \in \mathcal{M}$. The case when the noise model cannot be correctly described within the model set but where there exists a $\theta_{0} \in D_{\mathcal{M}}$ such that

$$
G\left(q, \theta_{0}\right)=G_{0}(q)
$$

will be denoted $G_{0} \in \mathcal{G}$.

Additional notation will be introduced when necessary in the sequel.

\section{Approaches to closed-loop identification}

It is important to realize that a directly applied prediction error method applied as if any feedback did not exist - will work well and give optimal accuracy if the true system can be described within the chosen model structure (i.e. if $\mathcal{S} \in \mathcal{M}$ ). Nevertheless, due to the pitfalls in closed-loop identification, several alternative methods have been suggested. One may distinguish between methods that

(a) Assume no knowledge about the nature of the feedback mechanism, and do not use $r$ even if known.

(b) Assume the regulator and the signal $r$ to be known (and typically of the linear form (2)).

(c) Assume the regulator to be unknown, but of a certain structure (like (2)).

If the regulator indeed has the form (2), there is no major difference between (a), (b) and (c): The noise-free relation (2) can be exactly determined based 
on a fairly short data record, and then $r$ carries no further information about the system, if $u$ is measured. The problem in industrial practice is rather that no regulator has this simple, linear form: Various delimiters, anti-windup functions and other non-linearities will have the input deviate from (2), even if the regulator parameters (e.g. PID-coefficients) are known. This strongly favors the first approach.

The methods for closed-loop identification correspondingly fall into the following main groups (see [6]):

(1) The Direct Approach: Apply a prediction error method and identify the open-loop system using measurements of the input $u$ and the output $y$.

(2) The Indirect Approach: Identify the closed-loop system using measurements of the reference signal $r$ and the output $y$ and use this estimate to solve for the open-loop system parameters using the knowledge of the controller.

(3) The Joint Input-Output Approach: Consider the input $u$ and the output $y$ jointly as the output from a system driven by the reference signal $r$ and noise. Use some method to determine the open-loop parameters from an estimate of this system.

In the following we will analyze several prediction error methods for closedloop identification. In particular we will study several schemes for indirect and joint input-output identification.

\section{The direct approach}

\subsection{General}

In the direct approach one typically works with models of the form

$$
y(t)=G(q, \theta) u(t)+H(q, \theta) e(t)
$$

The prediction errors for this model are given by

$$
\varepsilon(t, \theta)=H(q, \theta)^{-1}(y(t)-G(q, \theta) u(t))
$$

In general, the prediction error estimate is obtained as

$$
\hat{\theta}_{N}=\arg \min _{\theta} V_{N}(\theta)
$$


where typically

$$
V_{N}(\theta)=\frac{1}{2 N} \sum_{t=1}^{N} \varepsilon_{F}^{2}(t, \theta)
$$

Here $\varepsilon_{F}$ are the filtered prediction errors,

$$
\varepsilon_{F}(t, \theta)=L(q) \varepsilon(t, \theta)
$$

where $L$ is some stable prefilter. The effect of prefiltering is equivalent to changing the noise model to

$$
\bar{H}(q, \theta)=L^{-1}(q) H(q, \theta)
$$

Thus, in the analysis, we may assume $L \equiv 1$ without loss of generality.

The resulting estimates of the dynamics model and the noise model will be denoted $\hat{G}_{N}$ and $\hat{H}_{N}$,

$$
\hat{G}_{N}(q)=G\left(q, \hat{\theta}_{N}\right), \text { and } \hat{H}_{N}(q)=H\left(q, \hat{\theta}_{N}\right)
$$

The direct identification approach should be seen as the natural approach to closed-loop data analysis. The main reasons for this are

- It works regardless of the complexity of the regulator, and requires no knowledge about the character of the feedback.

- No special algorithms and software are required.

- Consistency and optimal accuracy is obtained if the model structure contains the true system (including the noise properties).

There are two drawbacks with the direct approach: One is that we will need good noise models. In open-loop operation we can use output error models (and other models with fixed or independently parameterized noise models) to obtain consistent estimates (but not of optimal accuracy) of $G$ even when the noise model $H$ is insufficient. See Theorem 8.4 in [10].

The second drawback is a consequence of this and appears when a simple model is sought that should approximate the system dynamics in a prespecified frequency norm. In open-loop we can do so with the output error method and a fixed prefilter/noise model that matches the specifications. For closed-loop data a prefilter/noise model that deviates from the true noise characteristics will introduce bias (cf. (9) below). The natural solution to this would be to first build a higher order model using the direct approach, with small bias, and then reduce this model to lower order with the proper frequency weighting. 
Another case that shows the necessity of good noise models concerns unstable systems. For closed-loop data, the true system to be identified could very well be unstable, although the closed-loop system naturally is stable. The prediction error methods require the predictor to be stable. This means that any unstable poles of $G$ must be shared by $H$, like in ARX, ARMAX and state-space models. Output error models cannot be used for this case. Just as in the open-loop case, models with common parameters between $G$ and $H$ require a consistent noise model for the $G$-estimate to be consistent.

\subsection{Bias distribution}

We shall now characterize in what sense the model approximates the true system, when it cannot be exactly described within the model class. This will be a complement to the open-loop discussion in Section 8.5 of [10].

Consider the model (5) where $G(q, \theta)$ is such that either $F_{y}(q)$ or $G(q, \theta)$ contains a delay. We have the prediction errors

$$
\begin{aligned}
\varepsilon(t) & =H^{-1}(q, \theta)(y(t)-G(q, \theta) u(t)) \\
& =H^{-1}(q, \theta)\left\{\left(G_{0}(q)-G(q, \theta)\right) u(t)+H_{0}(q) e(t)\right\} \\
& =H^{-1}(q, \theta)(\tilde{G}(q) u(t))+\left(H^{-1}(q, \theta) H_{0}(q)-1\right) e(t)+e(t) \\
& =H^{-1}(q, \theta)(\tilde{G}(q) u(t)+\tilde{H}(q) e(t))+e(t)
\end{aligned}
$$

Here

$$
\tilde{G}(q)=G_{0}(q)-G(q, \theta) \text { and } \tilde{H}(q)=H_{0}(q)-H(q, \theta)
$$

Insert (2) for $u$,

$$
\begin{aligned}
\varepsilon(t)=H^{-1}(q, \theta)\left\{\tilde { G } ( q ) \left(S_{0}(q)\right.\right. & r(t) \\
& \left.\left.-F_{y}(q) S_{0}(q) H_{0}(q) e(t)\right)+\tilde{H}(q) e(t)\right\}+e(t)
\end{aligned}
$$

Our assumption that the closed-loop system was well-defined implies that $\tilde{G} F_{y}$ contains a delay, as well as $\tilde{H}$ (since both $H$ and $H_{0}$ are monic). Therefore the last term of (6) is independent of the rest. Computing the spectrum of the first term we get (over-bar denotes complex conjugate)

$$
\begin{aligned}
\frac{1}{|H|^{2}}\left[\Phi_{u}|\tilde{G}|^{2}-2 \operatorname{Re} \tilde{H}^{*} \tilde{G} F_{y} S_{0} H_{0} \lambda_{0}+|\tilde{H}|^{2} \lambda_{0}\right] \\
=\frac{\Phi_{u}}{|H|^{2}}\left|\tilde{G}-\frac{\overline{F_{y} S_{0} H_{0}} \tilde{H} \lambda_{0}}{\Phi_{u}}\right|^{2}+\frac{\lambda_{0}|\tilde{H}|^{2}}{|H|^{2}}\left[1-\frac{\Phi_{u}^{e}}{\Phi_{u}}\right]
\end{aligned}
$$


Let us introduce the notation ( $B$ for "bias")

$$
B=\frac{\overline{F_{y} S_{0} H_{0}} \tilde{H} \lambda_{0}}{\Phi_{u}}
$$

then the spectral density of $\varepsilon$ becomes

$$
\Phi_{\varepsilon}=\frac{\Phi_{u}}{|H|^{2}}|\tilde{G}-B|^{2}+\frac{\lambda_{0}|\tilde{H}|^{2}}{|H|^{2}}\left[1-\frac{\Phi_{u}^{e}}{\Phi_{u}}\right]+\lambda_{0}
$$

Note that

$$
|B|^{2}=\frac{\lambda_{0}}{\Phi_{u}} \cdot \frac{\Phi_{u}^{e}}{\Phi_{u}} \cdot|\tilde{H}|^{2}
$$

The limiting model will minimize the integral of $\Phi_{\varepsilon}$, according to standard prediction error identification theory. We see that if $F_{y}=0$ (open-loop operation) we have $B=0$ and $\Phi_{u}^{e}=0$ and we re-obtain expressions that are equivalent to the expressions in Section 8.5 in [10].

Let us now focus on the case with a fixed noise model $H(q, \theta)=H_{*}(q)$. This case can be extended to the case of independently parameterized $G$ and $H$. Recall that any prefiltering of the data or prediction errors is equivalent to changing the noise model. The expressions below therefore contain the case of arbitrary prefiltering. For a fixed noise model, only the first term of (7) matters in the minimization, and we find that the limiting model is obtained as

$$
G_{\text {opt }}=\arg \min _{G} \int_{-\pi}^{\pi}\left|G_{0}-G-B\right|^{2} \frac{\Phi_{u}}{\left|H_{*}\right|^{2}} d \omega
$$

This is identical to the open-loop expression, except for the bias term $B$. Within the chosen model class, the model $G$ will approximate the biased transfer function $G_{0}-B$ as well as possible according the the weighted frequency domain function above. The weighting function $\Phi_{u} /\left|H_{*}\right|^{2}$ is the same as in the open-loop case. The major difference is thus that an erroneous noise model (or unsuitable prefiltering) may cause the model to approximate a biased transfer function. The expression (9) is a variant of the expression given in [11].

Let us comment the bias function $B$. First, note that while $G$ (in the fixed noise model case) is constrained to be causal and stable, the term $B$ need not be so. Therefore $B$ can be replaced by its stable, causal component (the "Wiener part") without any changes in the discussion. Next, from (8) we see that the bias-inclination will be small in frequency ranges where either (or all) of the following holds:

- The noise model is good ( $\tilde{H}$ is small).

- The feedback noise contribution to the input spectrum $\left(\Phi_{u}^{e} / \Phi_{u}\right)$ is small. 
- The signal to noise ratio is good $\left(\lambda_{0} / \Phi_{u}\right.$ is small $)$.

In particular, it follows that if a reasonably flexible, independently parameterized noise model is used, then the bias-inclination of the $G$-estimate can be negligible.

\subsection{Asymptotic variance expressions}

Let us now consider the asymptotic variance of the estimated transfer function $\hat{G}_{N}$ using the Asymptotic Black-Box theory of Section 9.4 in [10].

Note that the basic result

$$
\operatorname{Cov}\left[\begin{array}{l}
\hat{G}_{N} \\
\hat{H}_{N}
\end{array}\right] \sim \frac{n}{N} \Phi_{v}\left[\begin{array}{cc}
\Phi_{u} & \Phi_{u e} \\
\Phi_{u e}^{*} & \lambda_{0}
\end{array}\right]^{-1}
$$

applies also to the closed-loop case. Here $n$ is the model order, $N$ the number of data, and $\Phi_{u e}$ the cross spectrum between input $u$ and noise source $e$. From this general expression we can directly solve for the upper left element:

$$
\operatorname{Cov} \hat{G}_{N} \sim \frac{n}{N} \frac{\Phi_{v} \lambda_{0}}{\lambda_{0} \Phi_{u}-\left|\Phi_{u e}\right|^{2}}
$$

From (2) we easily find that

$$
\lambda_{0} \Phi_{u}-\left|\Phi_{u e}\right|^{2}=\lambda_{0}\left|S_{0}\right|^{2} \Phi_{r}=\lambda_{0} \Phi_{u}^{r}
$$

SO

$$
\operatorname{Cov} \hat{G}_{N} \sim \frac{n}{N} \frac{\Phi_{v}}{\Phi_{u}^{r}}
$$

The denominator of (11) is the spectrum of that part of the input that originates from the reference signal $r$. The open-loop expression has the total input spectrum here.

The expression (11) - which also is the asymptotic Cramer-Rao lower limit - tells us precisely "the value of information" of closed-loop experiments. It is the noise-to-signal ratio (where "signal" is what derives from the injected reference) that determines how well the open-loop transfer function can be estimated. From this perspective, that part of the input that originates from the feedback noise has no information value when estimating $G$. Since this property is, so to say, inherent in the problem, it should come as no surprise that several other methods for closed-loop identification can also be shown to 
give the same asymptotic variance, namely (11) (see, e.g., [5] and the results in Sections 5.5 and 6.6 below).

The expression (11) also clearly points to the basic problem in closed-loop identification: The purpose of feedback is to make the sensitivity function small, especially at frequencies with disturbances and poor system knowledge. Feedback will thus worsen the measured data's information about the system at these frequencies.

Note, though, that the "basic problem" is a practical and not a fundamental one: There are no difficulties, per se, in the closed-loop data, it is just that in practical use, the information contents is less. We could on purpose make closed-loop experiments with good information contents (but poor control performance).

Note that the output spectrum is, according to (3),

$$
\Phi_{y}=\left|G_{0}\right|^{2} \Phi_{u}^{r}+\left|S_{0}\right|^{2} \Phi_{v}
$$

The corresponding spectrum in open-loop operation would be

$$
\Phi_{y}^{\text {open }}=\left|G_{0}\right|^{2} \Phi_{u}+\Phi_{v}
$$

This shows that it may still be desirable to perform a closed-loop experiment: If we have large disturbances at certain frequencies we can reduce the output spectrum by $\left(1-\left|S_{0}\right|^{2}\right) \Phi_{v}$ and still get the same variance for $\hat{G}_{N}$ according to $(11)$.

Note that the basic result (11) is asymptotic when the orders of both $G$ and $H$ tend to infinity. Let us now turn to the case where the noise model is fixed, $H(q, \theta)=H_{*}(q)$. We will then only discuss the simple case where it is fixed to the true value

$$
H_{*}(q)=H_{0}(q)
$$

and where the bias in $\hat{G}_{N}$ is negligible. In that case the covariance matrix of $\hat{\theta}_{N}$ is given by the standard result

$$
\operatorname{Cov} \hat{\theta}_{N} \sim \frac{\lambda_{0}}{N}\left[\bar{E} \psi\left(t, \theta_{0}\right) \psi\left(t, \theta_{0}\right)^{T}\right]^{-1}
$$

where $\psi\left(t, \theta_{0}\right)$ is the negative gradient of

$$
\varepsilon(t, \theta)=\frac{1}{H_{*}(q)}(y(t)-G(q, \theta) u(t))
$$


evaluated at $\theta=\theta_{0}$. The covariance matrix is thus determined entirely by the second order properties (the spectrum) of the input, and it is immaterial whether this spectrum is a result of open-loop or closed-loop operation.

In particular we obtain in the case that the model order tends to infinity that

$$
\operatorname{Cov} \hat{G}_{N} \sim \frac{n}{N} \frac{\Phi_{v}}{\Phi_{u}}
$$

just as in the open-loop case.

\subsection{Asymptotic distribution of parameter estimates}

Consider again the model (5) and assume that the dynamics model and the noise model are independently parameterized, i.e. that

$$
G(q, \theta)=G(q, \rho) \text { and } H(q, \theta)=H(q, \beta)
$$

where $\rho$ and $\beta$ refers to the following partitioning of the parameter vector $\theta$ :

$$
\theta=\left[\begin{array}{l}
\rho \\
\beta
\end{array}\right]
$$

Also assume that $\mathcal{S} \in \mathcal{M}$ (i.e. that the true system is contained in the model set). Then, from the results in Section 9.3 in [10], we have the following: The covariance of the parameter estimate is

$$
\operatorname{Cov} \hat{\theta}_{N} \sim \frac{1}{N} P_{\theta}
$$

Here

$$
P_{\theta}=\lambda_{0} R_{\theta}^{-1}
$$

where $\lambda_{0}$ is the variance of the driving noise and

$$
R_{\theta}=\bar{E} \psi\left(t, \theta_{0}\right) \psi^{T}\left(t, \theta_{0}\right)
$$

We will in the following derive explicit expressions for $P_{\theta}$ in the case of a linear feedback regulator as in (2). The expressions will be given in the frequency domain. It will be convienient to consider the following augmented signal: Let

$$
\chi_{0}=\left[\begin{array}{l}
u \\
e
\end{array}\right]
$$


The spectrum of $\chi_{0}$ is

$$
\Phi_{\chi_{0}}=\left[\begin{array}{cc}
\Phi_{u} & \Phi_{u e} \\
\Phi_{u e}^{*} & \lambda_{0}
\end{array}\right]
$$

Since $\Phi_{u e}=-F_{y} S_{0} H_{0} \lambda_{0}$, we may also write this as

$$
\Phi_{\chi_{0}}=\Phi_{\chi_{0}}^{r}+\Phi_{\chi_{0}}^{e}
$$

where

$$
\Phi_{\chi_{0}}^{r}=\left[\begin{array}{cc}
\Phi_{u}^{r} & 0 \\
0 & 0
\end{array}\right] \text { and } \Phi_{\chi_{0}}^{e}=\lambda_{0}\left[\begin{array}{c}
F_{y} S_{0} H_{0} \\
-1
\end{array}\right]\left[\begin{array}{c}
F_{y} S_{0} H_{0} \\
-1
\end{array}\right]^{*}
$$

Using the frequency-domain results in Section 9.4 in [10] we see that $R_{\theta}$ can be written as

$$
R_{\theta}=\frac{1}{2 \pi} \int_{-\pi}^{\pi} \frac{1}{\left|H_{0}\right|^{2}} T_{\theta}^{\prime} \Phi_{\chi_{0}} T_{\theta}^{* *} d \omega
$$

where $T=[G H]$ and where $T_{\theta}^{\prime}=\left.\frac{d}{d \theta} T\right|_{\theta=\theta_{0}}$. From (14) it follows that

$$
T_{\theta}^{\prime} \Phi_{\chi_{0}} T_{\theta}^{* *}=T_{\theta}^{\prime} \Phi_{\chi_{0}}^{r} T_{\theta}^{*}+T_{\theta}^{\prime} \Phi_{\chi_{0}}^{e} T_{\theta}^{*}
$$

We may thus write

$$
R_{\theta}=R_{\theta}^{r}+R_{\theta}^{e}
$$

where $R_{\theta}^{r}$ is given by

$$
R_{\theta}^{r}=\frac{1}{2 \pi} \int_{-\pi}^{\pi} \frac{1}{\left|H_{0}\right|^{2}} T_{\theta}^{\prime} \Phi_{\chi_{0}}^{r} T_{\theta}^{\prime *} d \omega=\frac{1}{2 \pi} \int_{-\pi}^{\pi} \frac{\Phi_{u}^{r}}{\left|H_{0}\right|^{2}} G_{\theta}^{\prime} G_{\theta}^{\prime *} d \omega
$$

Note that $R_{\theta}^{r}$ only depends on $\Phi_{u}^{r}$ and not on the total input spectrum $\Phi_{u}$, as in the open-loop case. If we partition $R_{\theta}^{r}$ conformably with $\theta$ we see that, due to the chosen parameterization,

$$
R_{\theta}^{r}=\left[\begin{array}{cc}
R_{\rho}^{r} & 0 \\
0 & 0
\end{array}\right]
$$

where

$$
R_{\rho}^{r}=\frac{1}{2 \pi} \int_{-\pi}^{\pi} \frac{\Phi_{u}^{r}}{\left|H_{0}\right|^{2}} G_{\rho}^{\prime} G_{\rho}^{*} d \omega
$$


Returning to $R_{\theta}^{e}$ we see that

$$
R_{\theta}^{e}=\frac{1}{2 \pi} \int_{-\pi}^{\pi} \frac{1}{\left|H_{0}\right|^{2}} T_{\theta}^{\prime} \Phi_{\chi_{0}}^{e} T_{\theta}^{*} d \omega
$$

If we partition $R_{\theta}^{e}$ as

$$
R_{\theta}^{e}=\left[\begin{array}{cc}
R_{\rho}^{e} & R_{\rho \beta}^{e} \\
R_{\beta \rho}^{e} & R_{\beta}^{e}
\end{array}\right]
$$

we may write

$$
P_{\theta}=\lambda_{0}\left[\begin{array}{cc}
R_{\rho}^{r}+R_{\rho}^{e} & R_{\rho \beta}^{e} \\
R_{\beta \rho}^{e} & R_{\beta}^{e}
\end{array}\right]^{-1}
$$

The covariance of $\hat{\rho}_{N}$ is given by the top left block of $P_{\theta}$. It follows that

$$
\operatorname{Cov} \hat{\rho}_{N} \sim \frac{\lambda_{0}}{N}\left(R_{\rho}^{r}+\Delta\right)^{-1}
$$

where

$$
\Delta=R_{\rho}^{e}-R_{\rho \beta}^{e}\left(R_{\beta}^{e}\right)^{-1} R_{\beta \rho}^{e} \geq 0
$$

is the Schur complement of $R_{\beta}^{e}$ in the matrix $R_{\theta}^{e}$. Explicit expressions for $R_{\rho}^{e}$, $R_{\rho \beta}^{e}$ and $R_{\beta}^{e}$ can be derived using

$$
T_{\theta}^{\prime}\left[\begin{array}{c}
F_{y} S_{0} H_{0} \\
-1
\end{array}\right]=F_{y} S_{0} H_{0} G_{\theta}^{\prime}-H_{\theta}^{\prime}
$$

An important observation regarding the result (18) is that the term $\Delta$ is entirely due to the noise part of the input spectrum and since $\Delta \geq 0$ this contribution has a positive effect on the accuracy, contrarily to what one might have guessed. We conclude that in the direct method the noise in the loop is utilized in reducing the variance. Later we will see that for the indirect methods this contribution will be zero.

From (18) it is also clear that the worst-case experimental conditions - from the accuracy point of view - is when there is no external reference signal present, i.e. when $\Phi_{r}=0$. In that case

$$
\operatorname{Cov} \hat{\rho}_{N} \sim \frac{\lambda_{0}}{N} \Delta^{-1}
$$

Thus $\Delta$ characterizes the lower limit of achievable accuracy for the direct method. Now, if $\Delta$ is non-singular, we can consistently estimate the system 
parameters even though no reference signal is present. The exact conditions for this to happen are given in [12] for some common special cases. However, even if $\Delta$ is singular it will have a beneficial effect on the variance of the estimates, according to (18). Only when $\Delta=0$ there is no positive effect from the noise source on the accuracy of the estimates.

Let us study what makes $\Delta=0$ : According to (20), we can write

$$
\begin{aligned}
R_{\theta}^{e} & =\bar{E} \psi_{e}\left(t, \theta_{0}\right) \psi_{e}^{T}\left(t, \theta_{0}\right) & \psi_{e}\left(t, \theta_{0}\right) & =\left[\begin{array}{c}
L(q) w(t) \\
-H_{\beta}^{\prime}(q) w(t)
\end{array}\right] \\
w(t) & =H_{0}^{-1}(q) e(t) & L(q) & =F_{y}(q) S_{0}(q) H_{0}(q) G_{\rho}^{\prime}(q)
\end{aligned}
$$

Here the number of rows in $L$ and $H_{\beta}^{\prime}$ are consistent with the partitioning (17). From well known least-squares projections, we now recognize $\Delta$ as the error covariance matrix when estimating $L w$ from $H_{\beta}^{\prime} w$. If the noise model is very flexible, knowing $H_{\beta}^{\prime} w$ is equivalent to knowing all past $w$ (think, e.g., of $H$ being a FIR model of "almost infinite" length). Then $L w$ can be determined exactly from $H_{\beta}^{\prime} w$, and $\Delta=0$. At the other extreme, a fixed (and correct) noise model will make $\Delta=R_{\rho}^{e}=E L w(L w)^{T}$, which is the largest value $\Delta$ may have. This puts the finger on the value of information in the noise source $e$ for estimating the dynamics: It is the knowledge/assumption of a constrained noise model that improves the estimate of $G$. This also explains the difference between (11) (which assumes the noise model order to tend to infinity) and (12) (which assumes a fixed and correct noise model).

\section{The indirect approach}

\subsection{General}

Consider the linear feedback set-up (2). If the regulator $F_{y}$ is known and $r$ is measurable, we can use the indirect identification approach. It consists of two steps:

(1) Identify the closed-loop system from the reference signal $r$ to the output $y$.

(2) Determine the open-loop system parameters from the closed-loop model obtained in step 1, using the knowledge of the regulator.

It is clear that the main focus in the indirect approach is the identification of the closed-loop system. This can be advantageous. For instance, in connection to model-based control it is frequently pointed out that it is important 
that the model explains the closed-loop behavior of the plant as good as possible, correct modeling of the open-loop system is less critical, at least in some frequency ranges. Another advantage with the indirect approach is that any identification method can be applied in the first step, since estimating the closed-loop system $G_{c l}$ from measured $y$ and $r$ is an open-loop problem. Therefore methods like spectral analysis, instrumental variables, and subspace methods, that may have problems with closed-loop data, also can be applied. One drawback with closed-loop identification though, is that it is not clear, in general, how to perform the second step in an optimal way. In principle, we have to solve the equation (cf. (3))

$$
\hat{G}_{c l, N}=\frac{\hat{G}_{N}}{1+F_{y} \hat{G}_{N}}
$$

using the knowledge of the regulator. Typically, this gives an over-determined system of equations in the open-loop parameters which can be solved approximately in many ways (see, e.g., Section 5.7 below). The exact solution to (21) is of course

$$
\hat{G}_{N}=\frac{\hat{G}_{c l, N}}{1-F_{y} \hat{G}_{c l, N}}
$$

but this will lead to a high-order estimate $\hat{G}_{N}$ - typically the order of $\hat{G}_{N}$ will be equal to the order of $\hat{G}_{c l, N}$ plus the order of the regulator $F_{y}$. For methods, like the prediction error method, that allow arbitrary parameterizations $G_{c l}(q, \theta)$ it is natural to let the parameters $\theta$ relate to properties of the open-loop system $G$, so that in the first step we should use a model

$$
y(t)=G_{c l}(q, \theta) r(t)+H_{c l}(q, \theta) e(t)
$$

with

$$
G_{c l}(q, \theta)=\frac{G(q, \theta)}{1+F_{y}(q) G(q, \theta)}
$$

The identification method of applying a standard least-squares prediction error method to the model (22), (23) will henceforth be referred to as the indirect method even though it is really just a smart parameterization of the general indirect method.

Since identifying $G_{c l}$ in (22) is an open-loop problem consistency will not be lost if we choose a fixed noise model/prefilter $H_{c l}(q, \theta)=H_{c l, *}(q)$ to shape the bias distribution of $G_{c l}$ (cf. Section 4.2).

The parameterization can be arbitrary, and we shall comment on it below. It is quite important to realize that as long as the parameterization describes the same set of $G$, the resulting transfer function $\hat{G}$ will be the same, regardless 
of the parameterizations. The choice of parameterization may thus be important for numerical and algebraic issues, but it does not affect the statistical properties of the estimated transfer function.

\subsection{The dual-Youla method}

A nice and interesting idea is to use the so called dual-Youla parameterization that parameterizes all systems that are stabilized by a certain regulator $F_{y}$ (see, e.g., [18]). In the SISO case it works as follows. Let $F_{y}=X / Y(X, Y$ stable, coprime) and let $G_{n o m}=N / D(N, D$ stable, coprime) be any system that is stabilized by $F_{y}$. Then, as $R$ ranges over all stable transfer functions, the set

$$
\left\{G: G(q, \theta)=\frac{N(q)+Y(q) R(q, \theta)}{D(q)-X(q) R(q, \theta)}\right\}
$$

describes all systems that are stabilized by $F_{y}$. The unique value of $R$ that corresponds to the true plant $G_{0}$ is given by

$$
R_{0}=\frac{D\left(G_{0}-G_{n o m}\right)}{Y\left(1+F_{y} G_{0}\right)}
$$

This idea can now be used for identification (see, e.g., [7], [8], [16]): Given an estimate $\hat{R}_{N}$ of $R_{0}$ we can compute an estimate of $G_{0}$ as

$$
\hat{G}_{N}=\frac{N+Y \hat{R}_{N}}{D-X \hat{R}_{N}}
$$

Note that, using the dual-Youla parameterization we can write

$$
G_{c l}(q, \theta)=L(q) Y(q)(N(q)+Y(q) R(q, \theta))
$$

where $L=1 /(Y D+N X)$ is stable and inversely stable. With this parameterization the identification problem (22) becomes

$$
z(t)=R(q, \theta) x(t)+H_{c l}(q, \theta) e(t)
$$

where

$$
\begin{aligned}
& z(t)=y(t)-L(q) N(q) Y(q) r(t) \\
& x(t)=L(q) Y^{2}(q) r(t)
\end{aligned}
$$

Thus the dual-Youla method is a special parameterization of the general indirect method. This means, especially, that the statistical properties of the 
resulting estimates for the indirect method remain unaffected for the dualYoula method (cf. the discussion in the previous section). The main advantage of this method is of course that the obtained estimate $\hat{G}_{N}$ is guaranteed to be stabilized by $F_{y}$, which clearly is a nice feature.

Before turning to the statistical properties of the indirect approach, let us pause and study an interesting variant of the parameterization idea used in (23) which will provide useful insights into the connection between the direct and indirect methods.

\subsection{A formal connection between direct and indirect methods}

The noise model $H$ in a linear dynamics model structure has often turned out to be a key to interpretation of different "methods". The distinction between the models/"methods" ARX, ARMAX, output error, Box-Jenkins, etc, is entirely explained by the choice of the noise model. Also the practically important feature of prefiltering is equivalent to changing the noise model. Even the choice between minimizing one- or $k$-step prediction errors can be seen as a noise model issue. See, e.g., [10] for all this.

Therefore it should not come as a surprise that also the distinction between the fundamental approaches of direct and indirect identification can be seen as a choice of noise model.

The idea is to parameterize $G$ as $G(q, \theta)$ and $H$ as

$$
H(q, \theta)=H_{1}(q, \theta)\left(1+F_{y}(q) G(q, \theta)\right)
$$

We thus link the noise model to the dynamics model. There is nothing strange with that: So do ARX and ARMAX models. Note that this particular parameterization scales $H_{1}$ with the inverse model sensitivity function. Now, the predictor for

$$
y(t)=G(q, \theta) u(t)+H(q, \theta) e(t)
$$

is

$$
\hat{y}(t \mid \theta)=H^{-1}(q, \theta) G(q, \theta) u(t)+\left(1-H^{-1}(q, \theta)\right) y(t)
$$

Using $u=r-F_{y} y$ we get

$$
\hat{y}(t \mid \theta)=H_{1}^{-1}(q, \theta) \frac{G(q, \theta)}{1+F_{y}(q) G(q, \theta)} r(t)+\left(1-H_{1}^{-1}(q, \theta)\right) y(t)
$$


But this is exactly the predictor also for the model of the closed-loop system

$$
y(t)=G_{c l}(q, \theta) r(t)+H_{1}(q, \theta) e(t)
$$

with the closed-loop transfer function parameterized in terms of the openloop one, as in (23). The indirect approach to estimate the system in terms of the closed-loop model (27) is thus identical to the direct approach with the noise model (26). This is regardless of the parameterization of $G$ and $H_{1}$. Among other things, this shows that we can use any theory developed for the direct approach (allowing for feedback) to evaluate properties of the indirect approach.

\subsection{Bias distribution}

For a moment assume that $G_{c l}$ is estimated using a prediction error method with a fixed noise model/prefilter $H_{*}$ and that $G_{c l}$ is parameterized according to (23). Our model can thus be written

$$
y(t)=\frac{G(q, \theta)}{1+F_{y}(q) G(q, \theta)} r(t)+H_{*}(q) e(t)
$$

We then have the following result for the bias. We know that the limiting estimate $G_{o p t}$ is given by

$$
\begin{aligned}
G_{o p t} & =\arg \min _{G} \int_{-\pi}^{\pi}\left|\frac{G_{0}}{1+F_{y} G_{0}}-\frac{G}{1+F_{y} G}\right|^{2} \frac{\Phi_{r}}{\left|H_{*}\right|^{2}} d \omega \\
& =\arg \min _{G} \int_{-\pi}^{\pi}\left|\frac{G_{0}-G}{1+F_{y} G}\right|^{2} \frac{\left|S_{0}\right|^{2} \Phi_{r}}{\left|H_{*}\right|^{2}} d \omega
\end{aligned}
$$

Now, this is no clear cut minimization of the distance $G_{0}-G$. The estimate $G_{\text {opt }}$ will be a compromise between making $G$ close to $G_{0}$ and making $1 /\left(1+F_{y} G\right)$ (the model sensitivity function) small. There will thus be a "bias-pull" towards transfer functions that give a small sensitivity for the given regulator, but unlike (9) it is not easy to quantify this bias component. However, if the true system can be represented within the model set, this will always be the minimizing model, so there is no bias in this case, and, furthermore, the resulting estimate of the closed-loop system will always be the best least-squares approximation of the true closed-loop system with the frequency weighting $\Phi_{r} /\left|H_{*}\right|^{2}$.

As mentioned previously, from the statistical point of view the indirect method and the dual-Youla method are equivalent. This means, in particular, that the bias expression (28) is also valid for the dual-Youla method. To see this we first note that the dual-Youla method (25) applied with a fixed noise model 
$H_{*}$ gives the following characterization of the resulting $R$-estimate:

$$
R_{\text {opt }}=\arg \min _{R} \int_{-\pi}^{\pi}\left|R_{0}-R\right|^{2} \frac{\Phi_{x}}{\left|H_{*}\right|^{2}} d \omega
$$

Using (24) we get that

$$
\begin{aligned}
R_{0}-R & =\frac{D}{Y}\left[\frac{G_{0}-G_{n o m}}{1+F_{y} G_{0}}-\frac{G-G_{n o m}}{1+F_{y} G}\right] \\
& =\frac{D\left(1+F_{y} G_{n o m}\right)}{Y} \frac{\left(G_{0}-G\right)}{1+F_{y} G} \cdot S_{0} \\
& =\frac{1}{L Y^{2}} \frac{\left(G_{0}-G\right)}{1+F_{y} G} \cdot S_{0}
\end{aligned}
$$

Now, since $\Phi_{x}=|L|^{2}|Y|^{4} \Phi_{r}$ it follows that expression (28) characterizes the bias distribution for the dual-Youla method also. Similar equivalence results can be shown for the variance properties, to be studied next. The details will be left to the reader.

\subsection{Asymptotic variance expressions}

We will now turn to the variance properties of the indirect approach. According to the open-loop result, the asymptotic variance of $\hat{G}_{c l, N}$ will be

$$
\operatorname{Cov} \hat{G}_{c l, N} \sim \frac{n}{N} \frac{\Phi_{v, c l}}{\Phi_{r}}=\frac{n}{N} \frac{\left|S_{0}\right|^{2} \Phi_{v}}{\Phi_{r}}
$$

regardless of the noise model $H_{*}$. Here $\Phi_{v, c l}$ is the spectrum of the additive noise $v_{c l}$ in the closed-loop system (3), which equals the open-loop additive noise, filtered through the true sensitivity function. To transform this result to variance of the open-loop transfer function, we use Gauss' approximation formula

$$
\operatorname{Cov} \hat{G}_{N}=\frac{d G}{d G_{c l}} \operatorname{Cov} \hat{G}_{c l, N}{\frac{d G}{d G_{c l}}}^{*}
$$

It is easy to verify that

$$
\frac{d G}{d G_{c l}}=\frac{1}{S_{0}^{2}}
$$

SO

$$
\operatorname{Cov} \hat{G}_{N} \sim \frac{n}{N} \frac{\Phi_{v}}{\left|S_{0}\right|^{2} \Phi_{r}}=\frac{n}{N} \frac{\Phi_{v}}{\Phi_{u}^{r}}
$$

which - not surprisingly - equals what the direct approach gives, i.e. (11). 


\subsection{Asymptotic distribution of parameter estimates}

We will now apply the same analysis tools as we used in Section 4.4 for the direct method, to the indirect method.

\subsubsection{Independently parameterized noise model}

Consider the following model

$$
y(t)=G_{c l}(q, \rho) r(t)+H_{c l}(q, \beta) e(t)
$$

where, as in $(23), G_{c l}$ is parameterized in terms of the open-loop parameters. Estimating $\rho$ and $\beta$ in (29) is an open-loop problem. All standard open-loop results can thus be applied also in this case. As an example, assume $\mathcal{S} \in \mathcal{M}$, then we can immediately write down the expressions for the covariance of $\hat{\rho}$ :

$$
\operatorname{Cov} \hat{\rho}_{N} \sim \frac{\lambda_{0}}{N}\left(R_{c l}\right)^{-1}
$$

where

$$
R_{c l}=\frac{1}{2 \pi} \int_{-\pi}^{\pi} \frac{\Phi_{r}}{\left|H_{c l, 0}\right|^{2}} G_{c l, \rho}^{\prime} G_{c l, \rho}^{*} d \omega
$$

Note that $G_{c l, \rho}^{\prime}=S_{0}^{2} G_{\rho}^{\prime}$ and $\left|H_{c l, 0}\right|^{2}=\left|S_{0}\right|^{2}\left|H_{0}\right|^{2}$, hence

$$
R_{c l}=\frac{1}{2 \pi} \int_{-\pi}^{\pi} \frac{\Phi_{u}^{r}}{\left|H_{0}\right|^{2}} G_{\rho}^{\prime} G_{\rho}^{*} d \omega
$$

This is in fact identical to expression (16) and we conclude that, for the indirect method,

$$
\operatorname{Cov} \hat{\rho}_{N} \sim \frac{\lambda_{0}}{N}\left(R_{\rho}^{r}\right)^{-1}
$$

As we remarked before this covariance will always be larger than the covariance obtained in the direct method, equation (18). The difference stemming from the term $\Delta$ that is missing in (30). Thus, in terms of accuracy of the parameter estimates, the direct method outperforms the indirect.

\subsubsection{Fixed noise model}

Consider now the case where the noise model is fixed $H(q, \beta)=H_{*}(q)$. Typically $H_{*} \neq H_{0}$ so that we are in the situation $\mathcal{S} \notin \mathcal{M}$. The analysis above will thus not apply here. However, since estimating $\rho$ in $(29)$ with $H(q, \beta)=H_{*}(q)$ is an open-loop problem we can use the standard open-loop covariance results 
for the case of an inconsistent noise model. To this end, assume $G_{0} \in \mathcal{G}$. Then we get (cf. expression (9.55) in [10])

$$
\operatorname{Cov} \hat{\rho} \sim \frac{1}{N} R_{*}^{-1} Q_{*} R_{*}^{-1}
$$

where

$$
\begin{aligned}
& R_{*}=\frac{1}{2 \pi} \int_{-\pi}^{\pi} \frac{\Phi_{r}}{\left|H_{*}\right|^{2}} G_{c l, \rho}^{\prime} G_{c l, \rho}^{\prime *} d \omega=\frac{1}{2 \pi} \int_{-\pi}^{\pi} \frac{\left|S_{0}\right|^{2} \Phi_{u}^{r}}{\left|H_{*}\right|^{2}} G_{\rho}^{\prime} G_{\rho}^{*} d \omega \\
& Q_{*}=\frac{1}{2 \pi} \int_{-\pi}^{\pi} \frac{\Phi_{v, c l} \Phi_{r}}{\left|H_{*}\right|^{4}} G_{c l, \rho}^{\prime} G_{c l, \rho}^{\prime *} d \omega=\frac{1}{2 \pi} \int_{-\pi}^{\pi} \frac{\left|S_{0}\right|^{4} \Phi_{v} \Phi_{u}^{r}}{\left|H_{*}\right|^{4}} G_{\rho}^{\prime} G_{\rho}^{*} d \omega
\end{aligned}
$$

For all $H_{*}$,

$$
R_{*}^{-1} Q_{*} R_{*}^{-1} \geq \lambda_{0}\left(R_{\rho}^{r}\right)^{-1}
$$

with equality for $H_{*}=H_{c l, 0}=S_{0} H_{0}$. Thus we have the following ranking: The direct method gives better accuracy than the indirect noise model with an independently parameterized noise model which in turn gives better accuracy than the indirect method with a fixed noise model.

\subsection{Indirect identification with optimal accuracy}

In the previous section we saw that indirect identification gives worse accuracy than direct. This is not the case in general for indirect methods, as we will see presently. In the following we will review a result that was first derived in [13] (see also [6] and [14] for related results) namely that, under certain circumstances, indirect identification gives the same level of accuracy as direct identification.

\subsubsection{ARMAX modeling}

Suppose we identify the closed-loop system using an ARMAX model

$$
A_{c l}(q) y(t)=B_{c l}(q) r(t)+C_{c l}(q) e(t)
$$

Thus, with $\eta$ denoting the closed-loop parameter vector, the system dynamics is modeled as

$$
G_{c l}(q, \eta)=\frac{B_{c l}(q)}{A_{c l}(q)}
$$

while the noise model becomes

$$
H_{c l}(q, \eta)=\frac{C_{c l}(q)}{A_{c l}(q)}
$$


Also assume $\mathcal{S} \in \mathcal{M}$. Next, let the regulator be given by

$$
F_{y}(q)=\frac{X(q)}{Y(q)}
$$

where the polynomials $X$ and $Y$ are assumed coprime. Then, if we in the second step of the indirect scheme model the open-loop system as

$$
A(q) y(t)=B(q) u(t)+C(q) e(t)
$$

we get the following. From

$$
G_{c l}=\frac{G}{1+F_{y} G}
$$

and

$$
H_{c l}=\frac{H}{1+F_{y} G}
$$

it follows that we may solve for $A, B$ and $C$ in (cf. (21))

$$
\left\{\begin{array}{l}
A_{c l}=A Y+B X \\
B_{c l}=B Y \\
C_{c l}=C Y
\end{array}\right.
$$

Equation (31) may be interpreted as a system of linear equations in the openloop parameters $\theta$,

$$
\Gamma \theta=\bar{\eta}
$$

where $\Gamma$ is completely determined by $X$ and $Y$ and where $\bar{\eta}$ depends on the estimated closed-loop parameters $\hat{\eta}$ and $Y$. The exact definitions of $\Gamma$ and $\bar{\eta}$ are not important at this stage but explicit expressions can be found in Appendix A. The best unbiased estimate of $\theta$ is the Markov estimate,

$$
\hat{\theta}=\left[\Gamma^{T}(\operatorname{Cov} \bar{\eta})^{-1} \Gamma\right]^{-1} \Gamma^{T}(\operatorname{Cov} \bar{\eta})^{-1} \bar{\eta}
$$

which gives

$$
\operatorname{Cov} \hat{\theta}=\left[\Gamma^{T}(\operatorname{Cov} \bar{\eta})^{-1} \Gamma\right]^{-1}
$$

Now, by explicitly forming (32) and carrying out the algebra it can be shown that

$$
\operatorname{Cov} \hat{\theta} \sim \frac{\lambda_{0}}{N}\left[\bar{E} \psi\left(t, \theta_{0}\right) \psi\left(t, \theta_{0}\right)^{T}\right]^{-1}
$$


The proof is given in Appendix A. Here $\psi$ is the negative gradient of the open-loop prediction errors

$$
\varepsilon(t, \theta)=\frac{1}{C(q)}(A(q) y(t)-B(q) u(t))
$$

But (34) is equivalent to the open-loop expression (13). Thus, under certain circumstances, indirect identification gives the same accuracy as direct identification. This result can be transformed to the frequency domain using expression (15).

As will become more apparent below, the fact that the dynamics model and the noise model share the same poles is crucial for obtaining the same accuracy as with the direct approach.

\subsubsection{Independently parameterized noise model}

Suppose now that we in the first step in the indirect method use the following model with an independently parameterized noise model:

$$
\begin{aligned}
y(t) & =G_{c l}\left(q, \rho_{\eta}\right) r(t)+H_{c l}\left(q, \beta_{\eta}\right) e(t) \\
& =\frac{B_{c l}(q)}{F_{c l}(q)} r(t)+\frac{C_{c l}(q)}{D_{c l}(q)} e(t)
\end{aligned}
$$

This is also known as a Box-Jenkins model. We assume also in this case that $\mathcal{S} \in \mathcal{M}$. Let the open-loop model be

$$
\begin{aligned}
y(t) & =G\left(q, \rho_{\theta}\right) u(t)+H\left(q, \beta_{\theta}\right) e(t) \\
& =\frac{B(q)}{F(q)} r(t)+\frac{C(q)}{D(q)} e(t)
\end{aligned}
$$

To find the indirect estimate of $G_{0}$ we may solve for $B$ and $F$ in (cf. (31))

$$
\left\{\begin{array}{l}
B_{c l}=B Y \\
F_{c l}=F Y+B X
\end{array}\right.
$$

This system of equations may be written in form similar to (32):

$$
\tilde{\Gamma} \rho_{\theta}=\bar{\rho}_{\eta}
$$

The Markov estimate is

$$
\hat{\rho}_{\theta}=\left[\tilde{\Gamma}^{T}\left(\operatorname{Cov} \bar{\rho}_{\eta}\right)^{-1} \tilde{\Gamma}\right]^{-1} \tilde{\Gamma}^{T}\left(\operatorname{Cov} \bar{\rho}_{\eta}\right)^{-1} \bar{\rho}_{\eta}
$$


This time, however, we do not obtain the open-loop expression for the variance. Instead, as shown in appendix B, we get

$$
\operatorname{Cov} \hat{\rho}_{\theta} \sim \frac{1}{N}\left(R_{\rho}^{r}\right)^{-1}
$$

where $R_{\rho}^{r}$ is given by (16). We once again conclude that, with an independently parameterized noise model, this indirect method gives worse accuracy than the direct method. The difference is quantified by the term $\Delta$ (cf. (18)) which is missing in (37). So by using an independently parameterized noise model in the indirect method the noise does not contribute in reducing the variance as in the direct method and the indirect method using ARMAX models.

\subsection{Discussion}

The variance expressions (18), (30) etc. are asymptotic in $N$, the number of data samples. An interesting question is whether they are still valid when $N$ is finite and especially if the direct method always gives better accuracy than the indirect method also for finite $N$. The following example shows that there are situations where the direct method does not outperform the indirect, even though the variance for the direct method asymptotically is smaller than that of the indirect method.

Example 2 Consider the following set-up: The true system is

$$
y(t)=G_{0}(q) u(t)+H_{0}(q) e(t)
$$

where

$$
G_{0}(q)=\frac{1}{1-1.6 q^{-1}+0.89 q^{-2}}, H_{0}(q)=\frac{1-0.6 q^{-1}+0.09 q^{-2}}{1-1.6 q^{-1}+0.89 q^{-2}}
$$

and where $\{e(t)\}$ is a white-noise sequence with variance $\lambda_{0}$. The system is controlled with the pole-placement regulator

$$
F_{y}(q)=q^{-1}-0.8 q^{-2}
$$

The closed-loop system is

$$
\begin{aligned}
y(t) & =\frac{G_{0}(q)}{1+F_{y}(q) G_{0}(q)} r(t)+\frac{H_{0}(q)}{1+F_{y}(q) G_{0}(q)} e(t) \\
& =\frac{1}{1-0.6 q^{-1}+0.09 q^{-2}} r(t)+e(t)
\end{aligned}
$$


In order to identify $G_{0}$ the direct and the indirect methods were employed. The following model was used in the direct method:

$$
\begin{aligned}
y(t) & =G(q, \rho) u(t)+H(q, \beta) e(t) \\
& =\frac{b_{0}}{1+f_{1} q^{-1}+f_{2} q^{-2}} u(t)+\frac{1+c_{1} q^{-1}+c_{2} q^{-2}}{1+d_{1} q^{-1}+d_{2} q^{-2}} e(t)
\end{aligned}
$$

i.e. a seventh-order Box-Jenkins model. In the indirect method we used the following third-order output error model:

$$
\begin{aligned}
y(t) & =\frac{G(q, \rho)}{1+F_{y}(q) G(q, \rho)} r(t)+e(t) \\
& =\frac{b_{0}}{1+\left(f_{1}+b_{0}\right) q^{-1}+\left(f_{2}-0.8 b_{0}\right) q^{-2}} r(t)+e(t)
\end{aligned}
$$

The true parameter vector - to be estimated - is

$$
\rho_{0}=\left[\begin{array}{lll}
1 & -1.6 & 0.89
\end{array}\right]^{T}
$$

To check the accuracy of the $\rho$-estimates for the two methods we performed 500 Monte Carlo simulations and collected $N$ data samples in each run. This was done for two different values of $N, N=200$ and $N=2000$. In the simulations we used a white-noise reference with variance 1 . The variance of the additive noise was also chosen equal to unity, i.e. $\lambda_{0}=1$.

As an illustration of the simulation results we have plotted the estimated pole locations in Fig. 2 along with the true locations $0.8 \pm i 0.5$. Comparing the left

Direct method, $\mathrm{N}=200$ Direct method, $\mathrm{N}=2000$
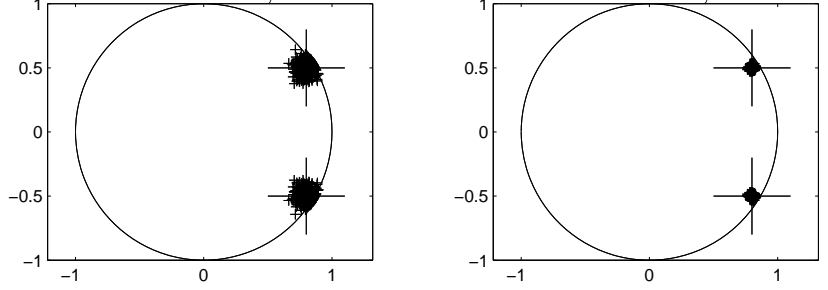

Indirect method, $\mathrm{N}=200$ Indirect method, $\mathrm{N}=2000$
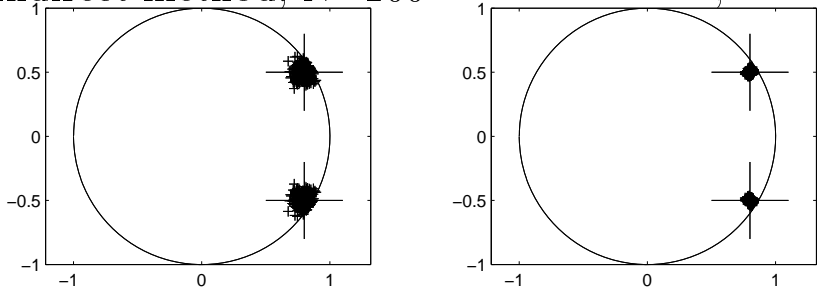

Fig. 2. Estimated pole locations. 
plots we see that for $N=200$ the direct and indirect methods roughly give the same variance. For $N=2000$ the direct method seems to give smaller variance though, just as theory predicts (cf. (18) and (30)). To verify these conjectures, we computed the sample averages

$$
\bar{\rho}_{N}=\frac{1}{500} \sum_{i=1}^{500} \hat{\rho}_{N}^{(i)}, \bar{P}_{N}=\frac{1}{499} \sum_{i=1}^{500}\left(\hat{\rho}_{N}^{(i)}-\bar{\rho}_{N}\right)\left(\hat{\rho}_{N}^{(i)}-\bar{\rho}_{N}\right)^{T}
$$

For the direct method we then obtained

$$
\begin{gathered}
\bar{\rho}_{200}^{(d)}=\left[\begin{array}{c}
1.0002 \\
-1.5989 \\
0.8931
\end{array}\right], \bar{P}_{200}^{(d)}=\frac{1}{200}\left[\begin{array}{ccc}
1.1033 & -0.4476 & 0.4279 \\
\times & 1.1249 & -0.9238 \\
\times & \times & 1.1229
\end{array}\right] \\
\bar{\rho}_{2000}^{(d)}=\left[\begin{array}{c}
0.9997 \\
-1.6000 \\
0.8900
\end{array}\right], \bar{P}_{2000}^{(d)}=\frac{1}{2000}\left[\begin{array}{ccc}
1.0125 & -0.4892 & 0.4794 \\
\times & 1.0804 & -0.9503 \\
\times & \times & 1.1171
\end{array}\right]
\end{gathered}
$$

The results for the indirect method were

$$
\begin{gathered}
\bar{\rho}_{200}^{(i d)}=\left[\begin{array}{c}
1.0014 \\
-1.5940 \\
0.8865
\end{array}\right], \bar{P}_{200}^{(i d)}=\frac{1}{200}\left[\begin{array}{ccc}
1.2131 & -0.3644 & 0.3061 \\
\times & 0.9779 & -0.7756 \\
\times & \times & 0.9388
\end{array}\right] \\
\bar{\rho}_{2000}^{(i d)}=\left[\begin{array}{c}
1.0018 \\
-1.6000 \\
0.8899
\end{array}\right], \bar{P}_{2000}^{(i d)}=\frac{1}{2000}\left[\begin{array}{ccc}
1.1871 & -0.4732 & 0.4518 \\
\times & 1.1942 & -1.1286 \\
\times & \times & 1.3992
\end{array}\right]
\end{gathered}
$$

A closer examination of these sample covariance matrices shows that, indeed,

$$
\bar{P}_{200}^{(d)}-\bar{P}_{200}^{(i d)}
$$

is indefinite while

$$
\bar{P}_{2000}^{(d)}-\bar{P}_{2000}^{(i d)}
$$

is negative definite. 
Thus for $N=200$ the direct method fail to give a smaller variance than indirect method. The main reason for this is that in the indirect method we estimate only three parameters, while in the direct method we estimate seven. Now, since $N$ is rather small the direct method will suffer from what is called "the curse of dimensionality", namely that the variance increases with the number of estimated parameters. Furthermore, in this particular example $\mathcal{S} \in \mathcal{M}$ for both methods (models). Thus the indirect method will not suffer from having an incorrect noise model which typically will be the case when using a fixed noise model. When increasing the sample size the direct method gradually gets better compared to the indirect and for $N=2000$ the variance of the direct method is smaller than that of the indirect, just as theory predicts.

\section{Joint input-output identification}

\subsection{General}

The third main approach to closed-loop identification is the so called joint input-output approach. We may rewrite (2) and (3) as

$$
\left[\begin{array}{l}
y(t) \\
u(t)
\end{array}\right]=\left[\begin{array}{c}
G_{0}(q) S_{0}(q) \\
S_{0}(q)
\end{array}\right] r(t)+\left[\begin{array}{cc}
S_{0}(q) H_{0}(q) & 0 \\
0 & -F_{y}(q) S_{0}(q) H_{0}(q)
\end{array}\right] e(t)
$$

Here the noise $e$ is vector-valued with covariance

$$
E e(t) e^{T}(t)=\Lambda_{0}=\left[\begin{array}{cc}
\lambda_{1} & \lambda_{12} \\
\lambda_{12} & \lambda_{2}
\end{array}\right]
$$

The case where $y$ and $u$ are measured in separate experiments with the same external reference applied corresponds to $\lambda_{12} \equiv 0$. If $y$ and $u$ were collected in the same experiment we would have $\lambda_{1}=\lambda_{12}=\lambda_{2}$. Thus (38) is rather general and comprises most practical situations.

The basic principle is now that once we have obtained estimates of the closedloop system $G_{c l, 0}=G_{0} S_{0}$ and the sensitivity function $S_{0}$ we could compute an estimate of $G_{0}$. Note that estimating $G_{c l, 0}$ and $S_{0}$ are open-loop problems so in principle all open-loop methods can be used (cf. the first step of the indirect approach). This idea really goes back to Akaike [1] who showed that in case

of closed-loop data one should proceed as follows if one were to use spectral 
analysis: Compute the spectral estimates

$$
\hat{G}_{c l, N}=\frac{\hat{\Phi}_{y x}^{N}}{\hat{\Phi}_{x}^{N}}
$$

and

$$
\hat{S}_{N}=\frac{\hat{\Phi}_{u x}^{N}}{\hat{\Phi}_{x}^{N}}
$$

where the signal $x$ is correlated with $y$ and $u$ but uncorrelated with the noise $e$ - a standard choice is $x=r$. The open-loop system may now be estimated as

$$
\hat{G}_{N}=\frac{\hat{G}_{c l, N}}{\hat{S}_{N}}=\frac{\hat{\Phi}_{y x}^{N}}{\hat{\Phi}_{u x}^{N}}
$$

Let us now discuss parametric solutions to this problem. Note that, just as in the indirect approach, computing an estimate of the open-loop system using estimates of closed-loop transfer functions, e.g., $G_{c l, 0}$ and $S_{0}$, can cause troubles. For instance, the straight-forward approach of dividing $\hat{G}_{c l, N}$ by $\hat{S}_{N}$, i.e.

$$
\hat{G}_{N}(q)=\frac{\hat{G}_{c l, N}(q)}{\hat{S}_{N}(q)}
$$

will lead to a high-order estimate of $G_{0}$ and, furthermore, the model order cannot be controlled. Typically the order of $\hat{G}_{N}$ will be the sum of the order of $\hat{G}_{c l, N}$ and the order of $\hat{S}_{N}$. To deal with this problem researchers have come up with various methods/parameterizations. One suggestion is the following.

\subsection{The coprime factor identification scheme}

The coprime factor identification scheme [17] can be understood as follows. Rewrite (38) using the filtered signal $x=F r$ :

$$
\left[\begin{array}{l}
y(t) \\
u(t)
\end{array}\right]=\left[\begin{array}{l}
N_{0, F}(q) \\
D_{0, F}(q)
\end{array}\right] x(t)+\left[\begin{array}{cc}
S_{0}(q) H_{0}(q) & 0 \\
0 & -F_{y}(q) S_{0}(q) H_{0}(q)
\end{array}\right] e(t)
$$

Here

$$
N_{0, F}=G_{0} S_{0} F^{-1} \text { and } D_{0, F}=S_{0} F^{-1}
$$


The choice of $F$ is discussed in [17] where it, e.g., is shown how $F$ should be chosen in order to make $N_{0, F}$ and $D_{0, F}$ normalized coprime. For our purposes here $F$ can be any stable, linear filter. Identifying $N_{0, F}$ and $D_{0, F}$ using measurements of $y, u$ and $x$ is still an open-loop problem, since $x$ and $e$ are uncorrelated. Typically, a standard prediction error method is used to find the estimates $\hat{N}_{N}$ and $\hat{D}_{N}$. Then the open-loop model is retrieved as

$$
\hat{G}_{N}(q)=\frac{\hat{N}_{N}(q)}{\hat{D}_{N}(q)}
$$

A benefit from using prediction error methods is that $N$ and $D$ can be parameterized on a common-denominator form:

$$
N(q)=\frac{b(q)}{f(q)}, D(q)=\frac{a(q)}{f(q)}
$$

This will give us control over the model order of $\hat{G}_{N}$ since then

$$
\hat{G}_{N}(q)=\frac{\hat{b}(q)}{\hat{a}(q)}
$$

\subsection{The two-stage method}

The next joint input-output method we will study is the two-stage method [15]. It is usually presented using the following two steps:

(1) Identify the sensitivity function $S_{0}$ using measurements of $u$ and $r$.

(2) Construct the signal $\hat{u}=\hat{S}_{N} r$ and identify the open-loop system as the mapping from $\hat{u}$ to $y$.

This method deserves a couple of remarks.

- Exact knowledge of the controller $F_{y}$ is not necessary since in the first step only the sensitivity function is modeled.

- In the first step a high-order model of $S_{0}$ can be used since we in the second step can control the open-loop model order independently.

- The simulated signal $\hat{u}$ will be the noise-free part of the input signal in the feedback system, thus $\hat{u}$ clearly is independent of the noise $e$.

The simplicity and robustness of the two-stage method makes it an attractive alternative to closed-loop identification. For our analysis it will be convienient to study the following reformulation of the two-stage method. This reformulation will also justify the classification of the two-stage method as a joint input-output method. 
Consider the following single input-two output model (cf. (38)):

$$
\left[\begin{array}{l}
y(t) \\
u(t)
\end{array}\right]=\left[\begin{array}{c}
G(q, \rho) S(\beta) \\
S(q, \beta)
\end{array}\right] r(t)+\left[\begin{array}{cc}
H_{1}(q) & 0 \\
0 & H_{2}(q)
\end{array}\right] e(t)
$$

As before we partition the parameter vector as

$$
\theta=\left[\begin{array}{l}
\rho \\
\beta
\end{array}\right]
$$

The prediction error for the model (39) is

$$
\varepsilon(t, \theta)=\left[\begin{array}{l}
\varepsilon_{y}(t, \theta) \\
\varepsilon_{u}(t, \theta)
\end{array}\right]
$$

where

$$
\varepsilon_{y}(t, \theta)=H_{1}^{-1}(q)(y(t)-G(q, \rho) S(q, \beta) r(t))
$$

and

$$
\varepsilon_{u}(t, \theta)=H_{2}^{-1}(q)(u(t)-S(q, \beta) r(t))
$$

Let

$$
W_{0}=\left[\begin{array}{cc}
w_{1}^{2} & 0 \\
0 & 1
\end{array}\right]
$$

and consider the weighted prediction error criterion

$$
V_{N}\left(\theta, w_{1}\right)=\frac{1}{2 N} \sum_{t=1}^{N} \varepsilon^{T}(t, \theta) W_{0}^{-1} \varepsilon(t, \theta)=\frac{1}{2 N} \sum_{t=1}^{N} w_{1}^{-2} \varepsilon_{y}^{2}(t, \theta)+\varepsilon_{u}(t, \theta)
$$

As $w_{1} \rightarrow \infty$ we obtain the limiting prediction error criterion

$$
V_{N}(\theta)=\lim _{w_{1} \rightarrow \infty} V_{N}\left(\theta, w_{1}\right)
$$

The claim is now: The resulting prediction error estimate for the two-stage method is

$$
\hat{\theta}_{N}=\arg \min _{\theta} V_{N}(\theta)
$$

This follows since, for large $w_{1}$ the $\beta$-parameters will minimize

$$
\frac{1}{2 N} \sum_{t=1}^{N} \varepsilon_{u}^{2}(t, \theta)=\frac{1}{2 N} \sum_{t=1}^{N}\left[H_{2}^{-1}(q)(u(t)-S(q, \beta) r(t))\right]^{2}
$$


regardless of the value of $\rho$, which then will minimize

$$
\frac{1}{2 N} \sum_{t=1}^{N} \varepsilon_{y}^{2}(t, \theta)=\frac{1}{2 N} \sum_{t=1}^{N}\left[H_{1}^{-1}(q)(y(t)-G(q, \rho) S(q, \beta) r(t))\right]^{2}
$$

Except for the additional weighting $w_{1}$ this is equivalent to the standard joint input-output method, given that we parameterize the model as in (39).

\subsection{The projection method}

We will now present another method for closed-loop identification that is inspired by Akaike's idea to correlate out the noise using the reference signal as instrumental variable. In form it will be similar to the two-stage method but the motivation for the methods will be quite different. The method will be referred to as the projection method. It can be understood as follows. In the first preliminary step we should "project" the input $u$ onto the reference $r$ using a non-causal, doubly infinite FIR-filter. This will result in a partitioning of the input signal into two orthogonal (uncorrelated) parts:

$$
u(t)=u_{\|}(t)+u_{\perp}(t)
$$

where

$$
u_{\|}(t)=S(q) r(t)=\lim _{M \rightarrow \infty} \sum_{k=-M}^{M} s_{k} r(t-k)
$$

Here it is assumed that $M$ does not grow faster than $N$ (the number of data), i.e.

$$
M \rightarrow \infty, N \rightarrow \infty, \text { and } \frac{M}{N} \rightarrow 0
$$

The basic relation

$$
y(t)=G_{0}(q) u(t)+v(t)
$$

can thus be rewritten as

$$
\begin{aligned}
y(t) & =G_{0}(q) u_{\|}(t)+w(t) \\
w(t) & =G_{0}(q) u_{\perp}(t)+v(t)
\end{aligned}
$$

The point is now that, since $w$ is uncorrelated with $r$ and hence uncorrelated with $u_{\|}$, any identification method can be applied to the constructed inputoutput pair $\left\{u_{\|}, y\right\}$. We can for instance apply a prediction error method to the model

$$
y(t)=G(q, \theta) u_{\|}(t)+H_{*}(q) e(t)
$$


Before studying an application example we would like to make the following remarks regarding the projection method:

- Here we chose to perform the projection using a non-causal filter but this step may also be performed non-parametrically as in Akaike's cross-spectral method.

- In practice $M$ can be chosen rather small. Good results are often obtained even with very modest values of $M$ (cf. Example 3 below).

- As stated here, the formulas for the projection method coincide with those of the two-stage method. This means, especially, that we may use the alternative interpretation (43) of the two-stage method also for the projection method, given that we parameterize $S$ as in (45). This will enable simultaneous analysis of the variance properties of the projection method and the two-stage method.

- For consistency of the projection method it is not required that the constructed signal $u_{\|}$equals $u$, i.e. $\hat{S}=S_{0}$ is not required, as is the case for the two-stage method (see (48) below). The crucial point is that $u_{\perp}$ is uncorrelated with $u_{\|}, u_{\perp} \equiv 0$ is not necessary.

- As a consequence of the previous remark it follows that the projection method can be applied to systems with non-linear elements such as limiters and anti-windup mechanisms in the loop, and still give consistent estimates, just as the direct method. There is one important difference though: The projection method gives consistent estimates regardless of the noise model, which may be fixed. This means that the projection method allows fitting the model to the data in a fixed, arbitrary, user-specified, frequencyweighted norm - a very attractive feature, especially in connection to identification for control. The price we pay for this flexibility is an increased variance due to the term $u_{\perp}$ in $w$.

- Finally, it would also be possible to project both the input $u$ and the output $y$ onto $r$ in the first step. This is in fact what Akaike suggested.

The robustness of the two-stage method was commented on before. In the following example we give an illustration of a situation where the closed-loop system contains a non-linear element. It is shown that in this case two-stage method fails to give an unbiased estimate but the projection method still gives the correct result.

Example 3 Consider the closed-loop system in Fig. 3. Here

$$
G_{0}(q)=q^{-1}-0.8 q^{-2}
$$

The feedback controller is

$$
\kappa(x)= \begin{cases}0.25 x+4, & \text { if } x>0 \\ 0.25 x-4, & \text { if } x<0\end{cases}
$$




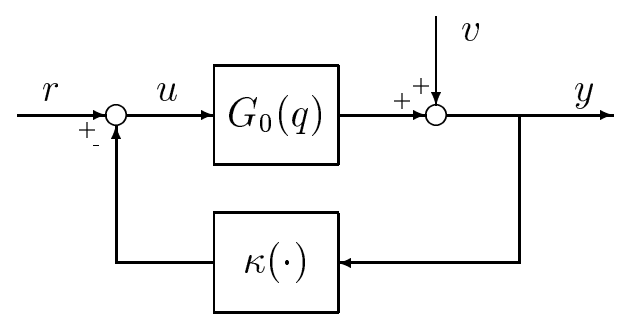

Fig. 3. Closed-loop system with non-linear feedback.

Thus, apart from just a scaling, the controller consists of a columbic friction element.

In order to identify $G$ a simulation was made with no noise present, i.e. with $v \equiv 0$, and using a white noise reference signal. 5000 data samples were collected. Thus the only errors in the models should be bias errors.

Next, the system was identified using the two-stage method and the projection method. The following models were employed in the first steps of the two-stage method:

(1) An 11th order FIR-model:

$$
S(q, \beta)=B(q)=b_{0}+b_{1} q^{-1}+\cdots+b_{1} 0 q^{-10}
$$

(2) An 11th order ARX-model:

$$
S(q, \beta)=\frac{B(q)}{A(q)}=\frac{b_{0}+b_{1} q^{-1}+\cdots+b_{4} q^{-5}}{1+a_{1} q^{-1}+\cdots+a_{6} q^{-6}}
$$

In the first step of the projection method the following model was used:

(1) An 11th order non-causal FIR-model:

$$
S(q)=\sum_{k=-5}^{5} s_{k} q^{-k}
$$

In the second step $G_{0}$ was estimated using $G(q, \rho)=B(q)=b_{1} q^{-1}-b_{2} q^{-2}$. Bode plots of the resulting models are shown in Fig. 4 together with a plot of $\left|G_{0}\right|$. As can be seen from the figure, the two-stage method gives biased estimates while the projection method gives an almost perfect one. Here we chose to use 11th order models in the first step of the algorithms, other choices give similar results as long as the model order is not too small (larger than 5 say). Especially, the projection method works fine with a finite number of parameters in the FIR-model.

For linear systems the two-stage method applied with a high-order FIR-model in the first step usually gives very good results. However, as this example shows, 


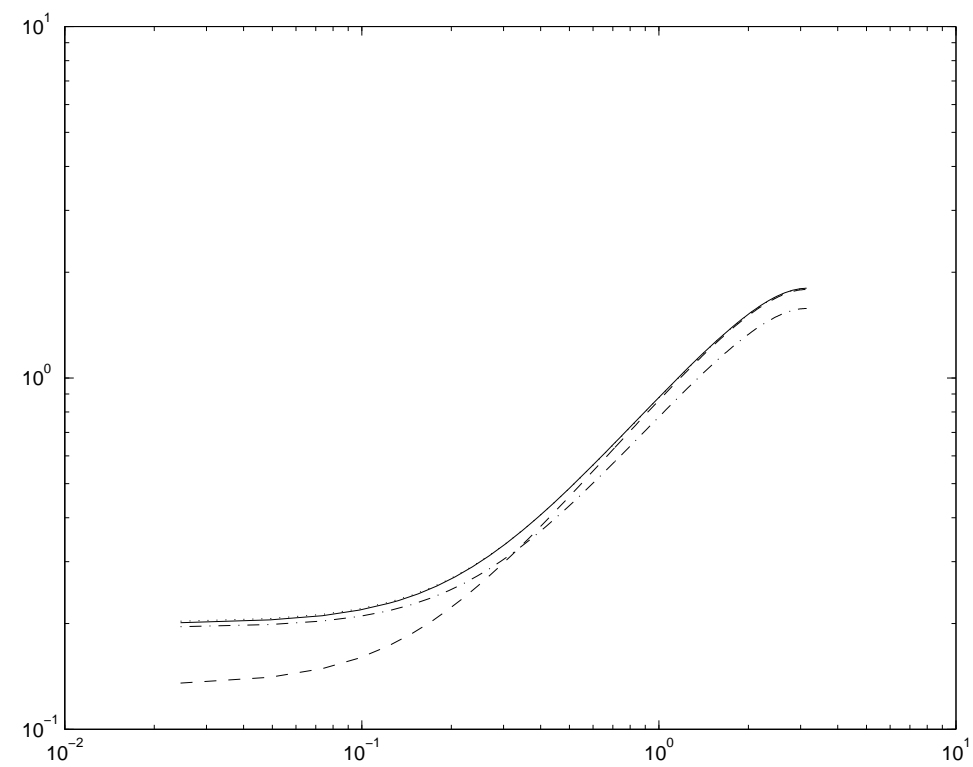

Fig. 4. Bode plot of the true system and the estimated models. True system (solid); Two-stage 1 (dashed); Two-stage 2 (dash-dotted); Projection (dotted).

the non-causal terms in the FIR-model in the projection method can not be excluded in general.

\subsection{Bias distribution}

\subsubsection{The coprime factor identification scheme}

The bias distribution for the coprime factor identification scheme, when applied with fixed noise models, will be characterized by

$$
\min _{N, D} \int_{-\pi}^{\pi}\left(\frac{\left|N_{0, F}-N\right|^{2}}{\left|H_{1}\right|^{2}}+\frac{\left|D_{0, F}-D\right|^{2}}{\left|H_{2}\right|^{2}}\right) \Phi_{x} d \omega
$$

With the filter $F$ tuned so as to make $N_{0, F}$ and $D_{0, F}$ normalized coprime, the coprime factor identification scheme ideally produces normalized coprime estimates $\hat{N}_{N}$ and $\hat{D}_{N}$ that, e.g., can be used in a subsequent control design step. In case of under-modeling, the noise models $H_{1}$ and $H_{2}$ can be used to shape the bias distribution in a control-relevant way.

\subsubsection{The two-stage method}

Suppose that we in the first step in the algorithm have obtained an estimate $\hat{S}_{N}$ of the sensitivity function. Then with a fixed noise model $H_{*}$ the model 
used in the second step is

$$
y(t)=G(q, \rho) \hat{S}_{N}(q) r(t)+H_{*}(q) e(t)
$$

The prediction errors become

$$
\begin{aligned}
\varepsilon(t, \rho) & =H_{*}^{-1}(q)\left(y(t)-G(q, \rho) \hat{S}_{N}(q) r(t)\right) \\
& =H_{*}^{-1}(q)\left\{\left(G_{0}(q) S_{0}(q)-G(q, \rho) \hat{S}_{N}(q)\right) r(t)+H_{0}(q) e(t)\right\}
\end{aligned}
$$

It follows that the resulting optimal $G$-estimate is given by

$$
G_{o p t}=\arg \min _{G} \int_{-\pi}^{\pi}\left|G_{0} S_{0}-G \hat{S}_{N}\right|^{2} \frac{\Phi_{r}}{\left|H_{*}\right|^{2}} d \omega
$$

Note that

$$
\left|G_{0} S_{0}-G \hat{S}_{N}\right|^{2}=\left|\left(G_{0}-G\right) S_{0}+G\left(S_{0}-\hat{S}_{N}\right)\right|^{2}
$$

Thus it is clear that for cases where $\hat{S}_{N} \neq S_{0}$ we will have a bias-pull towards models $G$ that minimize (48). On the other hand, if we in the first step have obtained a very accurate (high order) estimate of the sensitivity function $S_{0}$ this effect is negligible so that

$$
\left|G_{0} S_{0}-G \hat{S}_{N}\right|^{2} \approx\left|G_{0}-G\right|^{2}\left|S_{0}\right|^{2}
$$

Thus the mismatch $G_{0}-G$ will be minimized in a frequency dependent norm that is shaped by the true sensitivity function. It is also interesting to compare this result with the corresponding result for the indirect method, i.e. expression (28).

\subsubsection{The projection method}

Consider the model (46). Since the constructed signal $u_{\|}$is uncorrelated with the noise we immediately get (cf. expression (8.68) in [10])

$$
G_{\text {opt }}=\arg \min _{G} \int_{-\pi}^{\pi}\left|G_{0}-G\right|^{2} \frac{\Phi_{u_{\|}}}{\left|H_{*}\right|^{2}} d \omega
$$

Thus the limiting model $G_{o p t}$ is the best least-squares approximation of $G_{0}$, with the fixed frequency weighting

$$
W_{*}=\frac{\Phi_{u_{\|}}}{\left|H_{*}\right|^{2}}
$$

It is important to realize that this weighting is known to the user when applying the second step of the algorithm since $\Phi_{u_{\|}}$is is known. Furthermore by adjusting the design parameters $\Phi_{r}$ and $H_{*}$ it is possible to tune the weighting 
$W_{*}$ at will. Thus, with the projection method, the previously open question whether it is possible to construct a closed-loop identification method that allows arbitrary frequency weighting of the plant-model misfit has been answered affirmatively.

Finally, from (49) it is also clear that if $G_{0} \in \mathcal{G}$ then the resulting estimate will always be the correct one.

\subsection{Asymptotic variance expressions}

Applying the multivariable version of the standard result (10) (refer to [20] for details) to the situation (38) gives

$$
\operatorname{Cov}\left[\begin{array}{l}
\hat{N}_{N} \\
\hat{D}_{N}
\end{array}\right] \sim \frac{n}{N} \frac{\Phi_{w}}{\Phi_{r}}
$$

Here the signal $w$ is given by

$$
w(t)=\left[\begin{array}{cc}
S_{0}(q) H_{0}(q) & 0 \\
0 & -F_{y}(q) S_{0}(q) H_{0}(q)
\end{array}\right] e(t)
$$

Under the assumption that $y$ and $u$ were collected in the same experiment, i.e. that $\lambda_{1}=\lambda_{12}=\lambda_{2}=\lambda_{0}$, we have

$$
\operatorname{Cov}\left[\begin{array}{c}
\hat{N}_{N} \\
\hat{D}_{N}
\end{array}\right] \sim \frac{n}{N} \frac{\left|S_{0}\right|^{2} \Phi_{v}}{\Phi_{r}}\left[\begin{array}{cc}
1 & -F_{y}^{*} \\
-F_{y} & \left|F_{y}\right|^{2}
\end{array}\right]
$$

Now, since $G_{0}=N_{0} / D_{0}$ we get from Gauss' approximation formula

$$
\operatorname{Cov} \hat{G}_{N} \sim \frac{n}{N} \frac{\left|S_{0}\right|^{2} \Phi_{v}}{\Phi_{r}} \frac{1}{\left|D_{0}\right|^{2}}\left[1-\frac{N_{0}}{D_{0}}\right]\left[\begin{array}{cc}
1 & -F_{y}^{*} \\
-F_{y} & \left|F_{y}\right|^{2}
\end{array}\right]\left[\begin{array}{c}
1 \\
-\left(\frac{N_{0}}{D_{0}}\right)^{*}
\end{array}\right]
$$

Carrying out the algebra and using that $D_{0}=S_{0}$ we see that

$$
\begin{aligned}
& \frac{1}{\left|D_{0}\right|^{2}}\left[1-\frac{N_{0}}{D_{0}}\right]\left[\begin{array}{cc}
1 & -F_{y}^{*} \\
-F_{y} & \left|F_{y}\right|^{2}
\end{array}\right]\left[\begin{array}{c}
1 \\
-\left(\frac{N_{0}}{D_{0}}\right)^{*}
\end{array}\right]= \\
& =\frac{1}{\left|S_{0}\right|^{2}}\left(\left|1+F_{y} G_{0}\right|^{2}\right)=\frac{1}{\left|S_{0}\right|^{4}}
\end{aligned}
$$


Thus

$$
\operatorname{Cov} \hat{G}_{N} \sim \frac{n}{N} \frac{\Phi_{v}}{\left|S_{0}\right|^{2} \Phi_{r}}=\frac{n}{N} \frac{\Phi_{v}}{\Phi_{u}^{r}}
$$

just as in the previous cases (cf. (11)). Analogous calculations will show that this result holds for the coprime factor identification scheme for arbritrary choices of the filter $F$.

Furthermore, these calculations also show that the two-stage method and the projection method give the same asymptotic variance expression as the other closed-loop methods, namely (11). To see this consider the alternative interpretation of these methods provided by the criterion in (42). The weight $w_{1}$ used there can be seen as a scaling of the noise model: $H_{1} \rightarrow w_{1} H_{1}$ (cf. (40)). Now, since the result (50) in fact is independent of the noise model (openloop operation), it follows that the above calculations hold for the two-stage and the projection methods as well. It follows that (11) is valid also for these methods. Thus the fundamental expression (11) applies to all the direct, indirect and joint input-output methods considered in this paper which is natural since this expression reflects the information contents in the closed-loop data (cf. the discussion in Section 4.3).

As a final remark we mention that, if we in the joint input-output method collect the input and output data in two identical but independent experiments (i.e. if $\lambda_{12}=0, \lambda_{1}=\lambda_{2}=\lambda_{0}$ ), the following expression for the asymptotic variance results:

$$
\operatorname{Cov} \hat{G}_{N} \sim \frac{n}{N} \frac{\left(1+\left|G_{0} F_{y}\right|^{2}\right) \Phi_{v}}{\Phi_{r}}
$$

Clearly this is different from expression (11) so to achieve the Cramer-Rao bound (11) it is important to collect the input-output data in one experiment, we can not use two independent experiments.

\subsection{Asymptotic distribution of parameter estimates}

We will now derive covariance expressions for the parameter estimate $\hat{\rho}_{N}$ for the projection method. The expressions will provide useful insights in the accuracy aspects of the projection method although the relation to the corresponding results for the direct and indirect methods will not be obvious. However, since the results will be derived using standard open-loop identification theory, it will be straight-forward to compare the covariance expressions for the projection method with their open-loop counterparts. 
Recall that the true system may be rewritten as

$$
y(t)=G_{0}(q) u_{\|}(t)+w(t)
$$

where artificial noise signal $w$,

$$
w(t)=G_{0}(q) u_{\perp}(t)+v(t)
$$

is uncorrelated with $u_{\|}$. Consider the model (46):

$$
y(t)=G(q, \theta) u_{\|}(t)+H_{*}(q) e(t)
$$

and suppose that $G_{0} \in \mathcal{G}$. From expression (9.55) in [10], we have that

$$
\begin{aligned}
\text { Cov } \hat{\rho} & \sim \frac{1}{N} R^{-1} Q R^{-1} \\
R & =\frac{1}{2 \pi} \int_{-\pi}^{\pi} \frac{\Phi_{u_{\|}}}{\left|H_{*}\right|^{2}} G_{\theta}^{\prime} G_{\theta}^{* *} d \omega \\
Q & =\frac{1}{2 \pi} \int_{-\pi}^{\pi} \frac{\Phi_{w} \Phi_{u_{\|}}}{\left|H_{*}\right|^{4}} G_{\theta}^{\prime} G_{\theta}^{*} d \omega
\end{aligned}
$$

Thus the expression for the covariance is equivalent to the open-loop expression, except that $\Phi_{u}$ is replaced by $\Phi_{u_{\|}}$and $\Phi_{v}$ by $\Phi_{w}$. The partitioning (44) implies that

$$
\Phi_{u_{\|}}=\Phi_{u}-\Phi_{u_{\perp}}=\Phi_{u}\left[1-\frac{\Phi_{u_{\perp}}}{\Phi_{u}}\right]
$$

Thus, whenever $\Phi_{u_{\perp}} \neq 0$,

$$
\Phi_{u_{\|}}<\Phi_{u}
$$

Furthermore, in most practical cases we will also have

$$
\Phi_{w}>\Phi_{v}
$$

It is clear that for the projection method the signal to noise ratio will in general be worse than for open-loop identification $\left(\Phi_{u_{\|}} / \Phi_{w}<\Phi_{u} / \Phi_{v}\right)$, hence the variance will be larger.

If the feedback controller is indeed linear and given by (2) and the noise model $H_{*}$ is fixed to the true value $S_{0} H_{0}$ (this case easily extends to the case of an independently parameterized noise model), then

$$
\begin{aligned}
\Phi_{u_{\|}} & =\Phi_{u}^{r}=\left|S_{0}\right|^{2} \Phi_{r} \\
\Phi_{w} & =\Phi_{v, c l}=\left|S_{0}\right|^{2} \Phi_{v}
\end{aligned}
$$


It follows that

$$
\operatorname{Cov} \hat{\rho} \sim \frac{1}{N}\left[\frac{1}{2 \pi} \int_{-\pi}^{\pi} \frac{\Phi_{r}}{\Phi_{v}} G_{\theta}^{\prime} G_{\theta}^{*} d \omega\right]^{-1}
$$

which is equivalent to the open-loop expression (with $\Phi_{u}=\Phi_{r}$ ). Thus, under these special circumstances, the projection method gives the same accuracy as open-loop identification. This last result also applies to the two-stage method given that the sensitivity function is estimated without error in the first step of the algorithm.

\section{Conclusions}

We have attempted to give a status report on identification of closed loop systems. The basic issues, several of which are well known, can be summarized as follows:

- The basic problem with closed-loop data is that it typically has less information about the open-loop system - an important purpose of feedback is to make the closed-loop system insensitive to changes in the open-loop system.

- Several methods that give consistent estimates for open-loop data may fail when applied in a direct way to closed-loop data. This includes spectral and correlation analysis, the instrumental variable method, the subspace methods and output error methods with incorrect noise model.

- The direct approach, i.e. prediction error methods, applied in a direct fashion, with a noise model that can describe the true noise properties still gives consistent estimates and optimal accuracy. No knowledge of the feedback is required. This should be regarded as a prime choice of methods. The basic disadvantage is the necessity of using a complete noise model, and the loss of the possibility of using a custom-made frequency-domain fit by prefiltering (which is the same as a "fake noise model"). This disadvantage should perhaps not be overemphasized, since it is also present in the common practice of using ARMAX models for open-loop data.

- If the regulator mechanism is correctly known, indirect identification can be applied. This means that the closed-loop system is identified and the openloop dynamics is retrieved from that estimate, using the knowledge of the regulator. Indirect identification appears in various shapes, depending on the parameterization of the model of the closed-loop system. Its basic advantage is that the dynamics model $G$ can be correctly estimated even without estimating any noise model. The main drawback is that if the regulator description is not correct - e.g. due to non-linearities and other discrepancies - that error will directly affect the system model. It typically also gives worse accuracy than the direct approach. 
- Joint input-output identification means that the output and the input are both modeled as outputs of a system driven by the reference signal and by noise. The reference signal thus has to be measurable, but knowledge of the regulator is not necessary. The open-loop dynamics can the be retrieved from this joint input-output model. The joint input-output methods provide consistent estimates of $G$ even with fixed prefilters/noise models, just as the indirect methods, but give worse accuracy than the direct method.

- The projection method, which is a joint input-output method, allows fitting the model to the data in a fixed, arbitrary, user-specified, frequency-domain norm. The price we pay is an increased variance error.

\section{Acknowledgement}

The authors wish to thank Dr. Paul Van den Hof for inspiring discussions and his contributions in the derivation of the asymptotic variance results for the two-stage method.

\section{References}

[1] H. Akaike. Some Problems in the Application of the Cross-Spectral Method. In B. Harris, editor, Spectral Analysis of Time Series, pages 81-107. John Wiley \& Sons, 1967.

[2] B.D.O. Anderson and M. Gevers. Identifiability of linear stochastic systems operating under linear feedback. Automatica, 18(2):195-213, 1982.

[3] R. de Callafon, P. Van den Hof, and M. Steinbuch. Control Relevant Identification of a Compact Disc Pick-up Mechanism. In Proceedings of the 32nd Conference on Decision and Control, volume 3, pages 2050-2055, San Antonio,TX, 1993.

[4] M. Gevers. Towards a Joint Design of Identification and Control. In H. L. Trentelman and J. C. Willems, editors, Essays on Control: Perspectives in the Theory and its Applications, pages 111-151. Birkhäuser, 1993.

[5] M. Gevers, L. Ljung, and P. Van den Hof. Asymptotic variance expression for closed-loop identification and their relevance in identification for control. In Proceeding of the 11th IFAC Symposium on System Identification, Fukuoka, Japan, 1997.

[6] I. Gustavsson, L. Ljung, and T. Söderström. Identification of Processes in Closed Loop - Identifiability and Accuracy Aspects. Automatica, 13:59-75, 1977. 
[7] F. R. Hansen. A fractional representation to closed-loop system identification and experiment design. Phd thesis, Stanford University, Stanford, CA, USA, 1989.

[8] F. R. Hansen, G. F. Franklin, and R. Kosut. Closed-loop identification via the fractional representation: experiment desgin. In Proceedings of the American Control Conference, pages 1422-1427, Pittsburg, PA, 1989.

[9] W. S. Lee, B. D. O. Anderson, I. M. Y. Mareels, and R. L. Kosut. On some key issues in the windsurfer approach to adaptive robust control. Automatica, 31(11):1619-1636, 1995.

[10] L. Ljung. System Identification: Theory for the User. Prentice-Hall, 1987.

[11] L. Ljung. Information contents in identification data from closed loop operation. In Proc. 32nd IEEE Conference on Decision and Control, pages 2242-2247, San Antonio, TX, December 1993.

[12] T. Söderström, I. Gustavsson, and L. Ljung. Identifiability conditions for linear systems operating in closed loop. Int. J. Control, 21(2):234-255, 1975.

[13] T. Söderström, L. Ljung, and I. Gustavsson. On The Accuracy Of Identification And the Design Of Identification Experiments. Technical Report 7428, Department of Automatic Control, Lund Institute of Technology, Lund, Sweden, 1974.

[14] T. Söderström, P. Stoica, and B. Friedlander. An Indirect Prediction Error Method for System Identification. Automatica, 27:183-188, 1991.

[15] P. M. J. Van den Hof and R. J. P. Schrama. An Indirect Method for Transfer Function Estimation from Closed Loop Data. Automatica, 29(6):1523-1527, 1993.

[16] P. M. J. Van den Hof and R. J. P. Schrama. Identification and Control Closed-loop Issues. Automatica, 31(12):1751-1770, 1995.

[17] P. M. J. Van den Hof, R. J. P. Schrama, R. A. de Callafon, and O. H. Bosgra. Identification of normalized coprime factors from closed-loop experimental data. European Journal of Control, 1(1):62-74, 1995.

[18] M. Vidyasagar. Control System Synthesis: A Factorization Approach. MIT Press, Cambridge, Mass., 1985.

[19] Z. Zang, R. R. Bitmead, and M. Gevers. Iterative Weighted Least-squares Identification and Weighted LQG Control Design. Automatica, 31(11):15771594, 1995.

[20] Y.-C. Zhu. Black-box identification of MIMO transfer functions: asymptotic properties of prediction error models. Int. Journal of Adaptive Control and Signal Processing, 3:357-373, 1989. 
A Proof of (34)

Let

$$
\begin{aligned}
& A(q)=1+a_{1} q^{-1}+\cdots+a_{n_{a}} q^{-n_{a}} \\
& B(q)=b_{1} q^{-1}+\cdots+b_{n_{b}} q^{-n_{b}} \\
& C(q)=1+c_{1} q^{-1}+\cdots+c_{n_{c}} q^{-n_{c}}
\end{aligned}
$$

and similarly for the closed-loop polynomials. We can, without loss of generality, take the regulator polynomials to be

$$
\begin{aligned}
& X(q)=x_{0}+x_{1} q^{-1}+\cdots+x_{n_{x}} q^{-n_{x}} \\
& Y(q)=1+y_{1} q^{-1}+\cdots+y_{n_{y}} q^{-n_{y}}
\end{aligned}
$$

It follows that $\Gamma$ in (32) can be written (the partitioning refers to the parameters in the $A, B$ and $C$ polynomials)

$$
\Gamma=\left[\begin{array}{ccc}
\Gamma_{Y} & \Gamma_{X} & 0 \\
0 & \Gamma_{Y} & 0 \\
0 & 0 & \Gamma_{Y}
\end{array}\right]
$$

where

$$
\Gamma_{Y}=\left[\begin{array}{cccc}
1 & & \\
y_{1} & \ddots & \\
\vdots & \ddots & 1 \\
& & y_{1} \\
& & \vdots
\end{array}\right], \Gamma_{X}=\left[\begin{array}{ccc}
x_{0} & & \\
x_{1} & \ddots & \\
\vdots & \ddots & x_{0} \\
& & x_{1} \\
& & \vdots
\end{array}\right]
$$


while

$$
\bar{\eta}=\left[\begin{array}{c}
\hat{a}_{c l, 1}-y_{1} \\
\hat{a}_{c l, 2}-y_{2} \\
\vdots \\
\hat{b}_{c l, 1} \\
\hat{b}_{c l, 2} \\
\vdots \\
\hat{c}_{c l, 1}-y_{1} \\
\hat{c}_{c l, 2}-y_{2} \\
\vdots
\end{array}\right]
$$

We have (as in (33))

$$
\operatorname{Cov} \hat{\theta}=\left[\Gamma^{T}(\operatorname{Cov} \bar{\eta})^{-1} \Gamma\right]^{-1}
$$

However,

$$
\operatorname{Cov} \bar{\eta} \sim \frac{\lambda_{0}}{N}\left[\bar{E} \psi_{c l}\left(t, \eta_{0}\right) \psi_{c l}\left(t, \eta_{0}\right)^{T}\right]^{-1}
$$

where $\psi_{c l}$ is the negative gradient of

$$
\varepsilon_{c l}(t, \eta)=\frac{A_{c l}(q)}{C_{c l}(q)}\left(y(t)-\frac{B_{c l}(q)}{A_{c l}(q)} r(t)\right)
$$

which, when evaluated at $\eta=\eta_{0}$, becomes

$$
\begin{aligned}
& \psi_{c l}\left(t, \eta_{0}\right)=\frac{1}{C_{c l, 0}(q)} {\left[-y(t-1), \ldots,-y\left(t-n_{a_{c l}}\right),\right.} \\
&\left.r(t-1), \ldots, r\left(t-n_{b_{c l}}\right), e(t-1), \ldots, e\left(t-n_{c_{c l}}\right)\right]^{T}
\end{aligned}
$$

Thus

$$
\operatorname{Cov} \hat{\theta} \sim \frac{\lambda_{0}}{N}\left[\bar{E} \Gamma^{T} \psi_{c l}\left(t, \eta_{0}\right) \psi_{c l}\left(t, \eta_{0}\right)^{T} \Gamma\right]^{-1}
$$

But since

$$
\begin{aligned}
& \frac{Y(q)}{C_{c l, 0}(q)}=\frac{1}{C_{0}(q)} \text { and } \\
& \frac{X(q) y(t)}{C_{c l, 0}(q)}-\frac{Y(q) r(t)}{C_{c l, 0}(q)}=-\frac{1}{C_{0}(q)} u(t)
\end{aligned}
$$


we have

$$
\begin{aligned}
\Gamma^{T} \psi_{c l}\left(t, \eta_{0}\right)=\frac{1}{C_{0}(q)} & {\left[-y(t-1), \ldots,-y\left(t-n_{a}\right),\right.} \\
& \left.u(t-1), \ldots, u\left(t-n_{b}\right), e(t-1), \ldots, e\left(t-n_{c}\right)\right]^{T}
\end{aligned}
$$

It follows that

$$
\Gamma^{T} \psi_{c l}\left(t, \eta_{0}\right)=-\frac{d}{d \theta} \frac{A(q)}{C(q)}\left(y(t)-\frac{B(q)}{A(q)} u(t)\right)
$$

evaluated at $\theta=\theta_{0}$. Thus $\Gamma^{T} \psi_{c l}\left(t, \eta_{0}\right)=\psi\left(t, \theta_{0}\right)$ and hence we have

$$
\operatorname{Cov} \hat{\theta} \sim \frac{\lambda_{0}}{N}\left[\bar{E} \psi\left(t, \theta_{0}\right) \psi\left(t, \theta_{0}\right)^{T}\right]^{-1}
$$

which is (34).

\section{B Proof of (37)}

Let

$$
\begin{aligned}
& B(q)=b_{1} q^{-1}+\cdots+b_{n_{b}} q^{-n_{b}} \\
& F(q)=1+f_{1} q^{-1}+\cdots+f_{n_{f}} q^{-n_{f}} \\
& C(q)=1+c_{1} q^{-1}+\cdots+c_{n_{c}} q^{-n_{c}} \\
& D(q)=1+d_{1} q^{-1}+\cdots+d_{n_{d}} q^{-n_{d}}
\end{aligned}
$$

and similarly for the closed-loop polynomials and let $X$ and $Y$ be as in appendix A. $\tilde{\Gamma}$ and $\bar{\rho}_{\eta}$ in $(36)$ becomes

$$
\tilde{\Gamma}=\left[\begin{array}{cc}
\Gamma_{Y} & 0 \\
\Gamma_{X} & \Gamma_{Y}
\end{array}\right], \bar{\rho}_{\eta}=\left[\begin{array}{c}
\hat{b}_{c l, 1} \\
\hat{b}_{c l, 2} \\
\vdots \\
\hat{f}_{c l, 1}-y_{1} \\
\hat{f}_{c l, 2}-y_{2} \\
\vdots
\end{array}\right]
$$

Similar to the derivation in appendix A we then get

$$
\hat{\rho}_{\theta}=\left[\tilde{\Gamma}^{T}\left(\operatorname{Cov} \bar{\rho}_{\eta}\right)^{-1} \tilde{\Gamma}\right]^{-1}
$$


while

$$
\operatorname{Cov} \bar{\rho}_{\eta} \sim \frac{\lambda_{0}}{N}\left[\bar{E} \psi_{c l, \rho_{\eta}}\left(t, \eta_{0}\right) \psi_{c l, \rho_{\eta}}\left(t, \eta_{0}\right)^{T}\right]^{-1}
$$

Here $\psi_{c l, \rho_{\eta}}$ is the negative gradient of the closed-loop predictions errors,

$$
\begin{aligned}
\varepsilon_{c l}(t, \eta) & =\frac{1}{H_{c l}\left(q, \beta_{\eta}\right)}\left(y(t)-G_{c l}\left(q, \rho_{\eta}\right) r(t)\right) \\
& =\frac{1}{H_{c l}\left(q, \beta_{\eta}\right)}\left(y(t)-\frac{B_{c l}(q)}{F_{c l}(q)} r(t)\right)
\end{aligned}
$$

taken w.r.t. $\rho_{\eta}$, giving

$$
\begin{aligned}
\psi_{c l, \rho_{\eta}}\left(t, \eta_{0}\right)=\frac{1}{H_{c l, 0}(q) F_{c l, 0}(q)}[r(t-1) & , \ldots, r\left(t-n_{b_{c l}}\right), \\
& \left.-\frac{B_{c l, 0}(q)}{F_{c l, 0}(q)}\left(r(t-1), \ldots, r\left(t-n_{f_{c l}}\right)\right)\right]
\end{aligned}
$$

where $H_{c l, 0}=S_{0} H_{0}$. Now, since

$$
\begin{aligned}
& \frac{F_{c l, 0}(q) Y(q)-B_{c l, 0}(q) X(q)}{F_{c l, 0}^{2}(q)}=\frac{S_{0}^{2}(q)}{F_{0}(q)} \\
& \frac{B_{c l, 0}(q) Y(q)}{F_{c l, 0}^{2}(q)}=\frac{S_{0}^{2}(q)}{F_{0}(q)} \cdot \frac{B_{0}(q)}{F_{0}(q)}
\end{aligned}
$$

we get (after some calculations)

$$
\begin{aligned}
\tilde{\Gamma}^{T} \psi_{c l, \rho_{\eta}}\left(t, \eta_{0}\right)=\frac{S_{0}(q)}{H_{0}(q) F_{0}(q)}\left[r(t-1), \ldots, r\left(t-n_{b}\right),\right. & \\
& \left.-\frac{B_{0}(q)}{F_{0}(q)}\left(r(t-1), \ldots, r\left(t-n_{f}\right)\right)\right]^{T}
\end{aligned}
$$

SO

$$
\begin{aligned}
\tilde{\Gamma}^{T} \psi_{c l, \rho_{\eta}}\left(t, \eta_{0}\right) & =-\frac{S_{0}(q)}{H_{0}(q)} \cdot \frac{d}{d \rho_{\theta}}\left(y(t)-\frac{B(q)}{F(q)} r(t)\right) \\
& =\frac{S_{0}(q)}{H_{0}(q)} \cdot \frac{d}{d \rho_{\theta}} G\left(q, \rho_{\theta}\right) r(t)
\end{aligned}
$$

where the derivatives on the right should be evaluated at $\rho_{\theta}=\rho_{\theta, 0}$. Expression (37) follows immediately after switching to the frequency domain. 